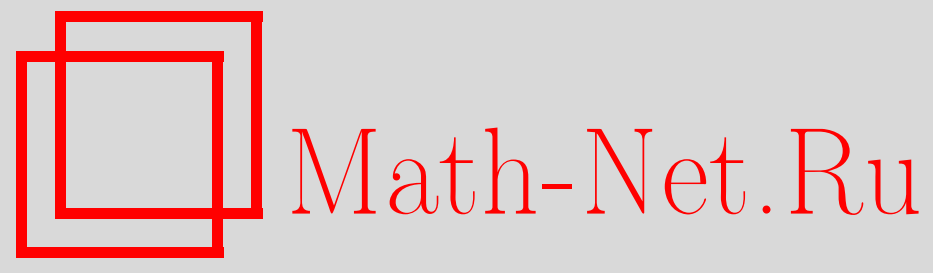

В. В. Палин, Геометрические решения задачи Римана для скалярного закона сохранения, Вестн. Сам. гос. техн. ун-та. Сер. Физ.-мат. науки, 2018, номер 4, 620-646

DOI: https://doi.org/10.14498/vsgtu1634

Использование Общероссийского математического портала MathNet.Ru подразумевает, что вы прочитали и согласны с пользовательским соглашением

http: //www . mathnet.ru/rus/agreement

Параметры загрузки:

IP : 54.209 .52 .79

26 апреля 2023 г., 13:10:09

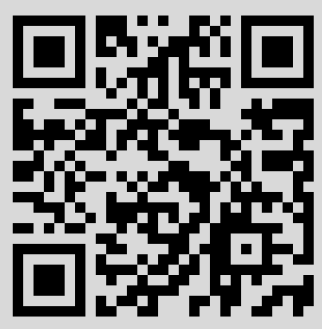


Вестн. Сам. гос. техн. ун-та. Сер. Физ.-мат. науки. 2018. Т. 22, № 4 . С. $620-646$ ISSN: 2310-7081 (online), 1991-8615 (print)

УДК 517.956.35

\title{
Геометрические решения задачи Римана для скалярного закона сохранения
}

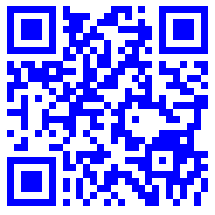

\section{В. В. Палин}

Московский государственный университет им. М. В. Ломоносова, механико-математический факультет, Россия, 119234, Москва, Ленинские Горы, 1.

\begin{abstract}
Аннотация
Для задачи Римана для скалярного закона сохранения предлагается новое определение решения, связанное с действием фазового потока ассоциированной гамильтоновой системы на начальную кривую. В первой части статьи выполняются подготовительные преобразования и дается основное определение. После этого предложенная схема определения решения обобщается на случай скалярного закона сохранения с функцией потока, зависящей от $x$. Во второй части статьи строится процедура выравнивания, позволяющая связать предлагаемое определение решения с решениями в смысле $D^{\prime}$. Доказывается корректность процедуры выравнивания (существование и единственность выравнивания для геометрического решения). Также в конце второй части статьи доказывается, что для случая закона сохранения с функцией потока, не зависящей от $x$, результат выравнивания геометрического решения в точности совпадает с единственным энтропийным решением.
\end{abstract}

Ключевые слова: задача Римана, законы сохранения, ассоциированная гамильтонова система.

Получение: 15 июля 2018 г. / Исправление: 11 ноября 2018 г. / Принятие: 12 ноября 2018 г. / Публикация онлайн: 27 ноября 2018 г.

\section{Научная статья}

(2) (7) Контент публикуется на условиях лицензии Creative Commons Attribution 4.0 International (https://creativecommons.org/licenses/by/4.0/deed.ru)

\section{Образец для цитирования}

Палин В. В. Геометрические решения задачи Римана для скалярного закона сохранения // Вестн. Сам. гос. техн. ун-та. Сер. Физ.-мат. науки, 2018. Т. 22, № 4. С. 620-646. doi: $10.14498 /$ vsgtu1634.

\section{Сведения об авторе}

Владимир Владимирович Палин (D) http://orcid.org/0000-0001-7529-7855

кандидат физико-математических наук; ассистент; каф. дифференциальных уравнений; e-mail: grey_stranger84@mail.ru 
Введение. Задача Римана для скалярного закона сохранения

$$
\left\{\begin{array}{l}
u_{t}+(\Phi(u))_{x}=0 \\
\left.u\right|_{t=0}=u_{-}+[u] \theta(x)
\end{array}\right.
$$

где $u_{-}$и $[u]$ - известные константы, $\theta(x)$ - функция Хевисайда, является хорошо изученной проблемой [1-3]. Стандартное определение решения такой задачи - в смысле интегрального тождества - широко известно (см., например, [4]). Известно также, что решение в смысле интегрального тождества не единственно, и стандартный подход к выбору решения заключается в наложении дополнительных условий на возможные решения (например, условие устойчивой ударной волны для решения задачи Римана [5,6]). Кроме того, существует определение обобщенного энтропийного решения этой задачи по Кружкову [8], причем обобщенное решение по Кружкову единственно.

Следует также отметить связанные с определением решения задачи Коши (1) определения и методы отыскания для обобщенного решения задачи Коши для уравнения Гамильтона-Якоби. Помимо стандартного определения слабого решения при помощи формулы Хопфа-Лакса [4,7], это определение минимаксного решения по Субботину [9] и идемпотентный подход, развитый в работах Маслова и соавторов [10,11].

В данной статье предлагается еще один вариант определения решения задачи Коши (1) и некоторого ее обобщения - геометрический. При этом в статье будет построено преобразование, позволяющее связать предлагаемое определение решения с разрывным решением в смысле интегрального тождества, и доказывается теорема существования и единственности геометрического решения. Следует отметить, что предлагаемое преобразование геометрического решения связано с известным в литературе правилом равных площадей [12], а точнее, является его обобщением на случай функции потока, зависящей от $x$. При этом предлагаемая конструкция применима не только к задаче Римана и позволяет описывать взаимодействие фронтов нескольких ударных волн. Задача о взаимодействии фронтов ранее изучалась при помощи специальной техники, например в [13], но предлагаемая в статье конструкция значительно упрощает нахождение решения.

\section{1. Определение геометрического решения.}

1.1. Ассоциированная гамилътонова система. Следуя О. А. Олейник [14], сделаем замену неизвестной функции в $(1)-$ положим $u=v_{x}$. Тогда уравнение примет вид

$$
v_{x t}+\left(\Phi\left(v_{x}\right)\right)_{x}=0,
$$

откуда после интегрирования по $x$ следует

$$
v_{t}+\Phi\left(v_{x}\right)=C_{1}(t)
$$

Заметим, что в силу того, что нас интересует только значение для $v_{x}$, мы можем считать, что $C_{1}(t)=0$. Действительно, замена

$$
\widetilde{v}=v-\int_{0}^{t} C_{1}(\tau) d \tau
$$


не меняет значения $v_{x}$, но обнуляет правую часть (2). Таким образом, мы поставили в соответствие уравнению в задаче (1) уравнение ГамильтонаЯкоби

$$
v_{t}+\Phi\left(v_{x}\right)=0
$$

Сразу сделаем обобщение: будем рассматривать случай гамильтониана, зависящего от $x$, т.е. $\Phi(p, x) \in C^{2}\left(\mathbb{R}^{2} ; \mathbb{R}\right)$. Тогда уравнение (3) примет вид

$$
v_{t}+\Phi\left(v_{x}, x\right)=0
$$

а соответствующая система характеристических ОДУ Гамильтона - вид

$$
\left\{\begin{array}{l}
\dot{x}=\frac{\partial \Phi}{\partial p} \\
\dot{p}=-\frac{\partial \Phi}{\partial x} .
\end{array}\right.
$$

Будем далее называть эту систему ассоциированной с законом сохранения

$$
u_{t}+(\Phi(u, x))_{x}=0
$$

Введем одно ограничение на гамильтониан $\Phi(p, x)$ :

$$
\exists R>0: \forall p \in \mathbb{R}, \forall x \in(-\infty ;-R) \cup(R ;+\infty): \frac{\partial \Phi}{\partial x}(p, x)=0 .
$$

1.2. Вспомогательные определения. Заметим, что если к ассоциированной гамильтоновой системе (5) добавить начальные условия

$$
\left.x\right|_{t=0}=x_{0},\left.\quad p\right|_{t=0}=p_{0},
$$

то в силу наложенного на гладкость $\Phi$ требования решение полученной задачи Коши существует и единственно для любой точки $\left(x_{0}, p_{0}\right)$ фазового пространства. Более того, т.к. гамильтониан $\Phi(p, x)$ - первый интеграл $(5)$, фазовое пространство разбивается на классы эквивалентности - кривые $l\left(x_{0}, p_{0}\right)$, вдоль которых гамильтониан постоянен. Обозначим через

$$
\left(X\left(t, x_{0}, p_{0}\right), P\left(t, x_{0}, p_{0}\right)\right)
$$

решение задачи Коши (5), (8). Тогда с гамильтоновой системой (5) можно связать однопараметрическое семейство операторов $S_{t}: \mathbb{R}^{2} \rightarrow \mathbb{R}^{2}$ сдвига вдоль траекторий, действующих по правилу

$$
S_{t}\left(x_{0}, p_{0}\right)=\left(X\left(t, x_{0}, p_{0}\right), P\left(t, x_{0}, p_{0}\right)\right) .
$$

В силу того, что теорема существования и единственности решения задачи Коши (5), (8) выполнена на всей фазовой плоскости, операторы $S_{t}$ сдвига вдоль траекторий образуют группу с операцией композиции.

1.3. Основное определение и связанные понятия. Возьмем $R>0$ из условия (7) и рассмотрим множество $\mathcal{K}_{R}$ непрерывных кривых $\gamma$ на фазовой плоскости таких, что

$$
\left\{\begin{array}{l}
\gamma \subset\left\{(x, p) \in \mathbb{R}^{2} \mid-R \leqslant p \leqslant R\right\} \\
\exists C_{-}(\gamma): \forall(x, p) \in \gamma, x<-R: p=C_{-}(\gamma), \\
\exists C_{+}(\gamma): \forall(x, p) \in \gamma, x>R: p=C_{+}(\gamma)
\end{array}\right.
$$


ОПредЕЛЕниЕ 1. Геометрическим решением закона сохранения (6) с начальной кривой $\gamma \in \mathcal{K}_{R}$ в момент времени $t$ назовем кривую $S_{t}(\gamma)$.

Заметим, что из того, что $S_{t}$ - группа операторов, сразу следует существование и единственность геометрических решений для заданной начальной кривой. Более того, так как задача Коши (5), (8) удовлетворяет всем условиям теоремы о непрерывности по параметру, в силу непрерывности начальной кривой получаем, что $S_{t}(\gamma)$ - непрерывная кривая для всех $t$.

ЗАмечАниЕ 1. Если начальная кривая $\gamma$-график некоторой функции $F_{\gamma}(x)$ и на некотором промежутке $x \in(a ; b)$ геометрическое решение остается графиком функции при всех $t \in(0 ; T)$, то геометрическое решение при $t \in(0 ; T), x \in(a ; b)$ совпадает с графиком классического решения задачи Коши для (6) с начальным условием $\left.u\right|_{t=0}=F_{\gamma}(x)$.

Доказательств о. Следует из того, что операторы сдвига вдоль траекторий в точности осуществляют сдвиг вдоль характеристик уравнения (4) и связи между этим уравнением и (6).

\section{2. Выравнивание кривой (связь со стандартным определением} энтропийного решения). В этом разделе мы построим связь между обобщенным энтропийным решением задачи Коши для (6) и геометрическим решением. Для этого мы опишем процедуру выравнивания кривой, которая ставит в соответствие геометрическому решению $S_{t}(\gamma)$ обобщенную функцию, и покажем, что полученная обобщенная функция является энтропийным решением.

2.1. Принципиалъная схема выравнивания кривой. Опишем процедуру построения кусочно-непрерывной функции $u(x)$ по кривой $S_{t}(\gamma)$. Для этого заметим, что кривая $S_{t}(\gamma)$ - многообразие, и она не является графиком функции, зависящей от $x$, если происходит опрокидывание фронта, т.е. возникает хотя бы одна точка $x_{0}$ такая, что в некоторой окрестности этой точки кривая $S_{t}(\gamma)$ пересекает прямые $x=$ const более чем в одной точке. Но тогда в некоторой окрестности каждой из точек пересечения $S_{t}(\gamma)$ с прямой $x=x_{0}$ эта кривая является графиком функции, зависящей от $p$. Возьмем любую из таких окрестностей. Ей можно поставить в соответствие связную и имеющую наибольшую длину окрестность $U=\left(q_{1}, q_{2}\right)$, обладающую тем же свойством. В этой окрестности кривая $S_{t}(\gamma)$ - график некоторой функции $\phi(p)$. При помощи описанной ниже процедуры выравнивания заменим $\phi(p)$ на монотонную непрерывную функцию $\psi(p)$. После этого сузим область определения функции $\psi(p)$ следующим образом: исключим все отрезки $\left[p_{1}, p_{2}\right]$ такие, что $\left.\psi(p)\right|_{\left[p_{1}, p_{2}\right]}=$ const. В результате получится функция $\psi_{*}(p)$, являющаяся строго монотонной. Если у $\psi_{*}(p)$ пустая область определения, то всей исходной окрестности $U$ соответствует разрыв $u(x)$ в точке $\psi\left(q_{1}\right)$. Иначе в силу строгой монотонности функции $\psi_{*}(p)$ соответствует кусочно-непрерывная обратная функция $u(x)=\psi_{*}^{-1}(x)$. Повторяя эту схему, в итоге получим кусочно-непрерывную функцию $u(x)$, соответствующую кривой $S_{t}(\gamma)$.

Перейдем теперь к описанию процедуры выравнивания кривой, являющейся графиком функции $\phi(p)$. 


\section{2. Определения локалъного и полного выравнивания в простей- шем случае.}

ОПреДЕЛЕНиЕ 2. Пусть $\phi(p) \in C([0,1])$. Точку $p_{0} \in(0,1)$ будем называть строгим локальным экстремумом функции $\phi(p)$, если найдется $\delta>0$ такое, что для всех $p_{1} \in\left(p_{0}-\delta, p_{0}\right), p_{2} \in\left(p_{0}, p_{0}+\delta\right)$ верно неравенство $\left(p_{1}-p_{0}\right)\left(p_{2}-p_{0}\right)<0$. Множество всех точек строгого локального экстремума будем обозначать $\mathcal{M}_{\phi}$.

ОПРЕДЕЛЕНИЕ 3. Введем класс функций $\mathcal{B}$ таких, что:

1) $\phi(p) \in C([0,1])$;

2) $\forall p \in(0,1): \phi(0)<\phi(p)<\phi(1)$;

3) отрезок $[0,1]$ можно представить в виде конечного объединения отрезкOB:

$$
[0,1]=\bigcup_{j=1}^{k}\left[m_{j-1}, m_{j}\right]
$$

так, что $m_{0}<m_{1}<\cdots<m_{k}$, и на каждом из отрезков $\left[m_{j-1}, m_{j}\right]$ функция $\phi(p)$ либо строго монотонна, либо постоянна.

ОПРеДЕЛЕНИЕ 4 . Полным выравниванием $\mathcal{E}_{*} \phi$ для $\phi \in \mathcal{B}$ назовем функцию из $\mathcal{B}$ такую, что найдутся $m \in(\mathbb{N} \cup\{0\})$ и отрезки $I_{k}=\left[p_{k}, q_{k}\right] \subseteq[0,1]$, $k=1, \ldots, m$ такие, что:

1) $\left.\mathcal{E}_{*} \phi\right|_{I_{k}}=C_{k} \in \mathbb{R}$ для всех $k=1, \ldots, m$;

2) $\forall p \in\left([0,1] \backslash \bigcup_{k=1}^{m} I_{k}\right): \mathcal{E}_{*} \phi(p)=\phi(p)$;

3) $\mathcal{E}_{*} \phi(p)$ монотонно неубывающая;

4) $\forall k \in\{1, \ldots, m\}: C_{k}=\frac{1}{q_{k}-p_{k}} \int_{p_{k}}^{q_{k}} \psi(\tau) d \tau$;

5) $\forall k \in\{1, \ldots, m\} \forall p \in\left(p_{k}, q_{k}\right): \int_{p_{k}}^{p} \phi(\tau) d \tau \geqslant C_{k}\left(p-p_{k}\right)$.

Целью этого и нескольких следующих пунктов является доказательство корректности определения полного выравнивания, т.е. доказательство следующей теоремы.

ТЕОРЕМА 1 (О КОРРЕКТНОСТИ ОПРЕДЕЛЕНИЯ ПОЛНОГО ВЫРАВНИВАНИЯ). ДЛЯ любой бункции $\phi(p) \in \mathcal{B}$ существует единственное полное выравнивание $\mathcal{E}_{*} \phi$.

Начнем с того, что определим локальное выравнивание. При этом для удобства дальнейшего изложения выделим требование, соответствующее пункту 5) определения полного выравнивания, в отдельное определение.

ОПределЕние 5. Локальным выравниванием для функции $\phi \in \mathcal{B}$ на отрезке $[\alpha, \beta]$ назовем функцию $\mathcal{E}_{[\alpha, \beta]} \phi \in \mathcal{B}$ такую, что:

1) $\forall p \in[\alpha, \beta]: \mathcal{E}_{[\alpha, \beta]} \phi(p)=\phi(\alpha)$;

2) $\forall p \in[0,1] \backslash[\alpha, \beta]: \mathcal{E}_{[\alpha, \beta]} \phi(p)=\phi(p)$;

3) $\phi(\alpha)=\frac{1}{\beta-\alpha} \int_{\alpha}^{\beta} \phi(\tau) d \tau$.

Заметим, что свойствам 1), 2) локального выравнивания соответствуют свойства полного выравнивания с теми же номерами; последнему свойству локального выравнивания соответствует свойство 4) полного выравнивания. 
Кроме того, непосредственно из определения локального выравнивания следует, что верно следующее простое утверждение.

ПРЕДЛОЖЕНИЕ 1. СВОЙСТВА ЛОКАЛЬНОГО ВЫРАВНИВАНИЯ. Справедливъ следующие утверждения.

1. При биксированных $\alpha, \beta$ локальное выравнивание $\mathcal{E}_{[\alpha, \beta]}$ применимо не ко всем $\phi \in \mathcal{B}$.

2. Ecлu $\left[\alpha_{1}, \beta_{1}\right] \cap\left[\alpha_{2}, \beta_{2}\right]=\emptyset, \operatorname{mo} \mathcal{E}_{\left[\alpha_{1}, \beta_{1}\right]} \mathcal{E}_{\left[\alpha_{2}, \beta_{2}\right]}=\mathcal{E}_{\left[\alpha_{2}, \beta_{2}\right]} \mathcal{E}_{\left[\alpha_{1}, \beta_{1}\right]}$.

3. Если $\left[\alpha_{1}, \beta_{1}\right] \subset\left[\alpha_{2}, \beta_{2}\right], \operatorname{mo} \mathcal{E}_{\left[\alpha_{2}, \beta_{2}\right]} \mathcal{E}_{\left[\alpha_{1}, \beta_{1}\right]}=\mathcal{E}_{\left[\alpha_{2}, \beta_{2}\right]}$.

4. Если $\phi \in \mathcal{B}, \mathcal{E}_{\left[\alpha, \beta_{1}\right]}$ u $\mathcal{E}_{\left[\beta_{1}, \beta\right]}$ применимы $\kappa \phi$, то $\mathcal{E}_{[\alpha, \beta]} \phi$ определено и $\mathcal{E}_{[\alpha, \beta]} \phi=\mathcal{E}_{\left[\alpha, \beta_{1}\right]} \mathcal{E}_{\left[\beta_{1}, \beta\right]} \phi$

5. Если $\mathcal{E}_{[\alpha, \beta]} \phi$ определено, $\lambda>0$, то $\mathcal{E}_{[\alpha, \beta]}(\lambda \phi)$ определено и $\mathcal{E}_{[\alpha, \beta]}(\lambda \phi)=$ $=\lambda \mathcal{E}_{[\alpha, \beta]} \phi$.

ОПредЕЛЕниЕ 6 . Локальное выравнивание $\mathcal{E}_{[\alpha, \beta]} \phi$ будем называть корректным, если

$$
\forall p \in(\alpha, \beta): \int_{\alpha}^{p} \phi(\tau) d \tau \geqslant \phi(\alpha)(p-\alpha) .
$$

Свойство корректности для локального выравнивания выполнено не всегда. Например, для функции

$$
\phi(x)=\left\{\begin{array}{cl}
5 x, & 0 \leqslant x \leqslant \frac{1}{5}, \\
\frac{3}{2}-\frac{5}{2} x, & \frac{1}{5} \leqslant x \leqslant \frac{2}{5} \\
\frac{5}{2} x-\frac{1}{2}, & \frac{2}{5} \leqslant x \leqslant \frac{3}{5} \\
\frac{5}{2}-\frac{5}{2} x, & \frac{3}{5} \leqslant x \leqslant \frac{4}{5} \\
5 x-\frac{7}{2}, & \frac{4}{5} \leqslant x \leqslant 1
\end{array}\right.
$$

локальное выравнивание $\mathcal{E}_{\left[\frac{3}{10}, \frac{7}{10}\right]}$ применимо, но не является корректным.

2.3. Лемма о непреръвном обходе. Для доказательства теоремы о корректности полного выравнивания важнейшую роль играют следующая техническая лемма и ее обобщения.

ЛЕмма 1 (о неПРеРЫвНом оБХоДЕ). Пусть $f(p), g(p) \in C([0 ; 1])$ maковъ, чmo:

1) $\forall p \in(0,1): f(0)<f(p)<f(1)$;

2) $\forall p \in(0,1): g(0)<g(p)<g(1)$;

3) $f(0)=g(0), f(1)=g(1)$;

4) отрезок $[0,1]$ можно представить в виде конечного объединения отрезков

$$
[0,1]=\bigcup_{j=1}^{k}\left[\mu_{j-1}, \mu_{j}\right]
$$

так, что $\mu_{0}<\mu_{1}<\cdots<\mu_{k}$, и на каждом из отрезков $\left[\mu_{j-1}, \mu_{j}\right]$ бункция $f(p)$ либо строго монотонна, либо постоянна; 
5) отрезок $[0,1]$ можно представить в виде конечного объединения отрезков

$$
[0,1]=\bigcup_{j=1}^{r}\left[\nu_{j-1}, \nu_{j}\right]
$$

так, что $\nu_{0}<\nu_{1}<\cdots<\nu_{r}$, и на каждом из отрезков $\left[\nu_{j-1}, \nu_{j}\right]$ функиия $g(p)$ либо строго монотонна, либо постоянна.

Тогда найдутся такие а $>0$ и функиии $h_{1}(t), h_{2}(t) \in C([0, a])$ такие, что $\forall t \in[0, a]: f\left(h_{1}(t)\right)=g\left(h_{2}(t)\right)$, причем $h_{1}(0)=h_{2}(0)=0, h_{1}(a)=h_{2}(a)=1$. Более того, если функиия $f(p)$ монотонно возрастает, то функиия $h_{2}(t)$ может бъть выбрана строго монотонной.

Доказательство.

1) Если хотя бы одна из функций $f(p), g(p)$ строго монотонно возрастает, то утверждение леммы очевидно.

Действительно, если $f(p)$ строго монотонно возрастает, то положим $a=1$, $h_{2}(t)=t, h_{1}(t)=g^{-1}(f(t))$. Заметим, что за исключением последнего пункта леммы (о строгой монотонности $\left.h_{2}(t)\right)$ функции $f(p)$ и $g(p)$ входят в утверждение леммы симметрично. Отсюда и из предыдущего утверждения следует, что если строго монотонной является $g(p)$, то утверждение леммы также верно.

2 ) Заметим, что аналогичное утверждению леммы утверждение верно для случая, когда $g(p)$ строго монотонно убывает, а неравенства в пункте 2) условий имеют другой знак, с той поправкой, что тогда $h_{2}(0)=1, h_{2}(a)=0$.

Действительно, если $g(p)$ строго монотонно убывает, то тогда $g(1-p)$ строго монотонно возрастает и работает первый пункт доказательства.

3) Так как сдвиг и растяжение - непрерывные преобразования, то утверждение верно с заменой в формулировке отрезка $[0,1]$ на произвольный отрезок $\left[p_{1}, p_{2}\right]$, а отрезка $[0, a]$ - на отрезок $\left[\tau_{1}, \tau_{2}\right]$.

4) Разобьем отрезок $[0,1]$ точками $0=p_{0}<p_{1}<\cdots<p_{k}=1$ по следующему правилу: потребуем, чтобы функция $f(p)$ на каждом из интервалов $\left(p_{j}, p_{j+1}\right)$ либо строго монотонно возрастала, либо строго монотонно убывала, либо была постоянной, причем для интервалов $\left(p_{j-1}, p_{j+1}\right)$ это условие бы не выполнялось. Нетрудно видеть, что такое разбиение отрезка $[0,1]$ для функции $f(p)$ с описанными свойствами существует и единственно.

5) Построим по разбиению из предыдущего пункта второе разбиение того же отрезка. Опишем алгоритм этого разбиения.

(i) Положим $q_{0}=0$. Далее возьмем $j=1$. Тогда $f\left(p_{j-1}\right)=g\left(q_{j-1}\right)$ по условию леммы.

(ii) Если $f(p)$ строго возрастает на $\left(p_{j-1}, p_{j}\right)$, то рассмотрим множество $Q_{j}$ точек $\tau>q_{j-1}$ таких, что $g(\tau)=f\left(p_{j}\right)$, и на интервале $\left(q_{j-1}, \tau\right)$ функция $g(q)$ удовлетворяет неравенству $g\left(q_{j-1}\right) \leqslant g(q) \leqslant g(\tau)$. В силу непрерывности функции $g(p)$ это множество непусто и замкнуто. Положим $q_{j}=\max Q_{j}$.

(iii) Если $f(p)$ строго убывает на $\left(p_{j-1}, p_{j}\right)$, то рассмотрим множество $Q_{j}$ точек $\tau<q_{j-1}$ таких, что $g(\tau)=f\left(p_{j}\right)$, и на интервале $\left(\tau, q_{j-1}\right)$ функция $g(q)$ удовлетворяет неравенству $g\left(q_{j-1}\right) \geqslant g(q) \geqslant g(\tau)$. В силу непрерывности функции $g(p)$ это множество непусто и замкнуто. Положим 


$$
q_{j}=\min Q_{j}
$$

(iv) Если $f(p)$ постоянна на интервале $\left(p_{j-1}, p_{j}\right)$, то положим $q_{j}=q_{j-1}$.

(v) Если $q_{j}<1$, то увеличим $j$ на единицу и перейдем к шагу (ii). Иначе алгоритм останавливается.

Заметим, что из неравенств $f(0)<f(p)<f(1)$ и конечности $k$ следует, что алгоритм остановится после конченого числа шагов.

6) Заметим теперь, что если $f(p)$ строго возрастает на $\left(p_{j-1}, p_{j}\right)$, то пара функций $\left.f\right|_{\left(p_{j-1}, p_{j}\right)}$ и $\left.g\right|_{\left(q_{j-1}, q_{j}\right)}$ удовлетворяет после аффинного преобразования условиям леммы с монотонной функцией $f$. Если же $f(p)$ строго убывает на $\left(p_{j-1}, p_{j}\right)$, то пара функций $\left.f\right|_{\left(p_{j-1}, p_{j}\right)}$ и $\left.g\right|_{\left(q_{j}, q_{j-1}\right)}$ удовлетворяет после аффинного преобразования второму пункту леммы с монотонной функцией $f$. Если же, наконец, $f(p)$ постоянна на $\left(p_{j-1}, p_{j}\right)$, то можно выбрать $h_{1}(t)$ линейной, значения которой монотонно возрастают от $p_{j-1}$ до $p_{j}$, и положить на соответствующем промежутке $h_{2}(t)=q_{j}$. Во всех случаях мы сможем построить пару функций $h_{1, j}(t), h_{2, j}(t)$, непрерывных и удовлетворяющих условиям леммы.

Осталось заметить, что благодаря третьему пункту доказательства мы можем выбрать области определения $\left[\tau_{j, 1}, \tau_{j, 2}\right]$ этих функций так, чтобы выполнялись равенства $\tau_{1,1}=0, \tau_{j, 2}=\tau_{j+1,1}$. Нетрудно видеть, что функции $h_{1}(t)$ и $h_{2}(t)$ такие, что $\left.h_{l}\right|_{\left[\tau_{j, 1}, \tau_{j, 2}\right]}=h_{l, j}, l=1,2, j \in\{1, \ldots, k\}$ - искомые.

Действительно, внутри каждого из интервалов они непрерывны по построению, а на концах соседних интервалов их значения совпадают благодаря равенствам $f\left(p_{j}\right)=g\left(q_{j}\right)$.

Из второго и третьего пунктов доказательства леммы о непрерывном обходе сразу следует, что ее можно обобщить следующим образом.

ЛЕММА 2 (ОБОБЩЕННАЯ ЛЕММА О НЕПРЕРЫВНОМ ОБХОДЕ). Пустъ функиนи $f(p) \in C\left(\left[\alpha_{1}, \beta_{1}\right]\right), g(p) \in C\left(\left[\alpha_{2}, \beta_{2}\right]\right)$ mаковиц, что:

1) глобальный максимум и глобальный минимум функиии $f(p)$ не могут лежать внутри интервала $\left(\alpha_{1}, \beta_{1}\right)$;

2) глобальный максимум и глобальный минимум функиии $g(p)$ не могут лежать внутри интервала $\left(\alpha_{2}, \beta_{2}\right)$;

3) либо

3а) $f\left(\alpha_{1}\right)=g\left(\alpha_{2}\right)$ u $f\left(\beta_{1}\right)=g\left(\beta_{2}\right)$, либо

3б) $f\left(\alpha_{1}\right)=g\left(\beta_{2}\right)$ u $f\left(\beta_{1}\right)=g\left(\alpha_{2}\right)$;

4) выполнены требования 4) и 5) леммы о непрерывном обходе с заменой отрезка $[0,1]$ на $\left[\alpha_{j}, \beta_{j}\right], j=1,2$.

Тогда найдутся такие $a>0$ и функиии $h_{1}(t), h_{2}(t) \in C([0, a])$ такие, что $\forall t \in[0, a]: f\left(h_{1}(t)\right)=g\left(h_{2}(t)\right)$, причем $h_{1}(0)=\alpha_{1}, h_{2}(0)=\alpha_{2}, h_{1}(a)=\beta_{1}$, $h_{2}(a)=\beta_{2}$ в случае $\left.3 \mathrm{a}\right), h_{1}(0)=\beta_{1}, h_{2}(0)=\alpha_{2}, h_{1}(a)=\alpha_{1}, h_{2}(a)=\beta_{2}$ в случае 3б).

\section{4. Существование локального выравнивания.}

Лемма 3. Для любой функиии $\phi \in \mathcal{B}$, имеющей хотя бы одну точку строгого локального минимума на $(0,1)$, существуют $\alpha, \beta \in(0,1)$ такие, что $\mathcal{E}_{[\alpha, \beta]}$ применимо к $\phi$, причем $\mathcal{E}_{[\alpha, \beta]} \phi \neq \phi$.

Доказательство. Так как $\phi \in \mathcal{B}$, из существования точки строгого локального минимума на $(0,1)$ следует существование точки локального 


\section{максимума.}

Пусть $p_{*}$ - точка локального максимума такая, что $\left|p_{*}\right|>0$ - наименьшее. Тогда $\phi(p)$ строго монотонно возрастает на $\left(0, p_{*}\right)$. Кроме того, в силу определения $\mathcal{B}$ верно неравенство $\phi\left(p_{*}\right)<\phi(1)$. Следовательно, в силу непрерывности $\phi(p)$ множество $\left\{p \in\left(p_{*}, 1\right) \mid \phi(p)=\phi\left(p_{*}\right), \exists q \in\left(p_{*}, p\right): \phi(q)<\phi\left(p_{*}\right)\right\}$ непусто.

Положим $p_{2}=\min \left\{p \in\left(p_{*}, 1\right) \mid \phi(p)=\phi\left(p_{*}\right), \exists q \in\left(p_{*}, p\right): \phi(q)<\phi\left(p_{*}\right)\right\}$. Далее в силу условия $\exists q \in\left(p_{*}, p\right): \phi(q)<\phi\left(p_{*}\right)$ функция $\phi(p)$ имеет хотя бы одну точку минимума на $\left[p_{0}, p_{2}\right]$ и значение в этой точке меньше, чем $\phi\left(p_{*}\right)$. Следовательно, корректно определены точки

$$
\begin{gathered}
p_{0}=\min \left\{p \in\left(p_{*}, p_{2}\right) \mid \phi(p)\right\}=\min \left\{\phi(q) \mid q \in\left[p_{*}, p_{2}\right]\right\}, \\
p_{1}=\max \left\{p \in\left(0, p_{*}\right) \mid \phi(p)=\phi\left(p_{0}\right)\right\} .
\end{gathered}
$$

Применим теперь обобщенную лемму о непрерывном обходе (лемма 2) к функции $\phi(p)$ на отрезках $\left[p_{1}, p_{*}\right]$ и $\left[p_{0}, p_{2}\right]$. Тогда найдутся отрезок $[0, a]$ и функции $h_{1}(t), h_{2}(t) \in C([0, a])$ такие, что $h_{1}(t) \in\left[p_{1}, p_{*}\right], h_{2}(t) \in\left[p_{0}, p_{2}\right]$, причем $\phi\left(h_{1}(t)\right)=\phi\left(h_{2}(t)\right)$.

Рассмотрим теперь

$$
I(t)=\int_{h_{1}(t)}^{h_{2}(t)}\left(\phi(\tau)-\phi\left(h_{1}(t)\right)\right) d \tau .
$$

Заметим, что $I(t) \in C([0, a])$,

$$
I(0)=\int_{p_{1}}^{p_{0}}\left(\phi(\tau)-\phi\left(p_{0}\right)\right) d \tau>0
$$

так как $\phi(\tau) \geqslant \phi\left(p_{0}\right)$ для всех $\tau \in\left(p_{1}, p_{2}\right)$. Кроме того,

$$
I(a)=\int_{p_{*}}^{p_{2}}\left(\phi(\tau)-\phi\left(p_{2}\right)\right) d \tau<0
$$

так как $\phi\left(p_{2}\right)=\phi\left(p_{*}\right) \geqslant \phi(\tau)$ для всех $\tau \in\left(p_{*}, p_{2}\right)$. Отсюда по теореме Вейерштрасса найдется $t_{*} \in[0, a]$ такое, что $I\left(t_{*}\right)=0$. Но тогда, выбирая $\alpha=h_{1}\left(t_{*}\right)$, $\beta=h_{2}\left(t_{*}\right)$, получаем, что $\phi(\alpha)=\phi(\beta)$,

$$
\int_{\alpha}^{\beta} \phi(\tau) d \tau=(\beta-\alpha) \phi(\alpha),
$$

т.е. $\mathcal{E}_{[\alpha, \beta]}$ применимо к $\phi$, причем $\mathcal{E}_{[\alpha, \beta]} \phi$ - постоянная функция на отрезке $[\alpha, \beta]$, в то время как $\phi(p)$ - не постоянна на этом отрезке.

Заметим, что приведенную выше лемму можно обобщить следующим обpaзом.

Лемма 4. Для любой функиии $\phi \in \mathcal{B}$ такой, что существуют $\mu, \nu \in(0,1)$ такие, что $\phi(p)$ строго монотонно убъвает на $(\mu, \nu)$, найдутся $\alpha, \beta \in(0,1)$ такие, что $\mathcal{E}_{[\alpha, \beta]}$ применимо $к \phi$, причем $\mathcal{E}_{[\alpha, \beta]} \phi \neq \phi$. 
До ка з ательст в о. Покажем, как модифицировать в этом случае доказательство леммы 3. По условию леммы, функция $\phi(p)$ не является монотонно неубывающей на $[0,1]$. Рассмотрим множество $G_{1} \subset(0, \mu]$ значений $q$ таких, что $\phi(p)$ монотонно не убывает на $[0, q]$. Так как $\phi(p)$ непрерывна и $\phi(1)>\phi(0)$, множество $G_{1} \neq \emptyset$ и замкнуто.

Пусть $g_{1}=\max G_{1}$. Далее, множество $\left\{q \in\left[0, g_{1}\right] \mid \phi(q)=\phi\left(g_{1}\right)\right\}$ непусто и замкнуто. Положим $p_{*}=\min \left\{q \in\left[0, g_{1}\right] \mid \phi(q)=\phi\left(g_{1}\right)\right\}$. Далее доказательство леммы 3 может быть повторено без изменений.

ЗАмечание 2. Для функции $\mathcal{E}_{[\alpha, \beta]} \phi(p)$, полученной в результате применения леммы 4 к $\phi(p)$, количество максимальных интервалов, при сужении на которые она монотонно убывает, уменьшилось на единицу. Применяя эту лемму к функции $\phi(p) \in \mathcal{B}$ конечное число раз, можно получить монотонно неубывающую функцию $\widetilde{\phi}(p)$. В свою очередь, функция $\widetilde{\phi}(p)$ удовлетворяет всем свойствам полного выравнивания, кроме свойства корректности.

2.5. Пример отсутствия единственности без условия корректности. Как показывает замечание 2 , для любой $\phi \in \mathcal{B}$ существует монотонно неубывающая $\widetilde{\phi}$, удовлетворяющая всем свойствам полного выравнивания (определение 4), кроме условия корректности. Как показывает приведенный далее пример, такая функция $\widetilde{\phi}$ определена не единственным образом, что вызывает трудности при обсуждении вопроса о единственности решения.

Действительно, рассмотрим функцию

$$
\phi(p)=\left\{\begin{array}{cc}
4 p, & 0 \leqslant p<\frac{1}{10}, \\
p+\frac{3}{10}, & \frac{1}{10} \leqslant p<\frac{2}{10}, \\
\frac{9}{10}-2 p, & \frac{2}{10} \leqslant p<\frac{3}{10} \\
p, & \frac{3}{10} \leqslant p<\frac{7}{10} \\
\frac{21}{10}-2 p, & \frac{7}{10} \leqslant p<\frac{8}{10}, \\
p-\frac{3}{10}, & \frac{8}{10} \leqslant p<\frac{9}{10} \\
4 p-3, & \frac{9}{10} \leqslant p \leqslant 1 .
\end{array}\right.
$$

Тогда функции

$$
\psi_{1}(p)=\left\{\begin{array}{cl}
4 p, & 0 \leqslant p<\frac{1}{10}, \\
\frac{4}{10}, & \frac{1}{10} \leqslant p<\frac{4}{10}, \\
p, & \frac{4}{10} \leqslant p<\frac{6}{10}, \\
\frac{6}{10}, & \frac{6}{10} \leqslant p<\frac{9}{10}, \\
4 p-3, & \frac{9}{10} \leqslant p \leqslant 1
\end{array}\right.
$$

$$
\psi_{2}(p)=\left\{\begin{array}{cl}
4 p, & 0 \leqslant p<\frac{1}{10}, \\
p+\frac{3}{10}, & \frac{1}{10} \leqslant p<\frac{2}{10}, \\
\frac{1}{2}, & \frac{2}{10} \leqslant p<\frac{8}{10}, \\
p-\frac{3}{10}, & \frac{8}{10} \leqslant p<\frac{9}{10}, \\
4 p-3, & \frac{9}{10} \leqslant p \leqslant 1
\end{array}\right.
$$


удовлетворяют всем условиям определения 4, кроме условия корректности.

2.6. Леммы о корректном выравнивании. Заметим, что полное выравнивание для $\phi \in \mathcal{B}$ строится как результат последовательного применения локальных выравниваний к функции $\phi$. Покажем, что на каждом шаге мы можем выбирать полное выравнивание, удовлетворяющее условию корректности. Для этого обратимся к доказательству леммы 3 и заметим, что мы не можем, вообще говоря, утверждать, что точка $t_{*}$ единственна. Воспользовавшись этим фактом, покажем, что среди доступных вариантов выбора точки $t_{*}$ всегда есть вариант, соответствующий корректному выравниванию. Для этого докажем сначала два вспомогательных утверждения.

ЛЕММА 5 (о КОРРЕКТНОСТИ КРАТЧАЙШЕГО ПУТИ ВЛЕВО). Пусть $\phi \in \mathcal{B}$ удовлетворяет условиям леммы 4. Пусть также $p_{1}, p_{*}, p_{0}, p_{2}-$ точки, описанные в доказательстве леммы 4, функиии $\chi_{j}(t) \in C([0, \widetilde{a}])$ таковь, что

$$
\begin{gathered}
\chi_{1}(0)=p_{1}, \quad \chi_{1}(\widetilde{a})=p_{*}, \quad \chi_{2}(0)=p_{*}, \quad \chi_{2}(\widetilde{a})=p_{0}, \\
\forall t \in[0, \widetilde{a}]: \phi\left(\chi_{1}(t)\right)=\phi\left(p_{*}+p_{0}-\chi_{2}(t)\right),
\end{gathered}
$$

$\chi_{2}(t)$ - монотонно возрастающая функция.

Обозначим

$$
\mathcal{I}(s, t)=\int_{\chi_{1}(t)}^{p_{*}+p_{0}-\chi_{2}(s)}\left(\phi(\tau)-\phi\left(\chi_{1}(t)\right)\right) d \tau
$$

Пусть также множество $\left\{t_{c r} \in(0, \widetilde{a}) \mid \mathcal{I}\left(t_{c r}, t_{c r}\right)\right\}$ непусто, $u$

$$
t_{*}=\max \left\{t_{c r} \in(0, \widetilde{a}) \mid \mathcal{I}\left(t_{c r}, t_{c r}\right)=0\right\} .
$$

Тогда для всех $s \in\left(t_{*}, \widetilde{a}\right]$ имеет место неравенство

$$
\mathcal{I}\left(s, t_{*}\right) \geqslant 0 \text {. }
$$

ЗАмечАниЕ 3. Существование функций $\chi_{j}(t)$, описанных в условиях предыдущей леммы, следует из обобщенной леммы о непрерывном обходе (лемма 2).

ЗАмечАниЕ 4. Заметим, что равенство $\mathcal{I}\left(t_{c r}, t_{c r}\right)=0$ эквивалентно тому, что локальное выравнивание $\mathcal{E}_{\left[\chi_{1}\left(t_{c r}\right), p_{*}+p_{0}-\chi_{2}\left(t_{c r}\right)\right]}$ применимо к $\phi$, а неравенство (9) эквивалентно тому, что для этого неравенства выполнено условие корректности (определение 6).

Доказательство леммы 5. Предположим противное. Пусть для некоторого $s_{0} \in\left(t_{*}, \widetilde{a}\right]$ верно противоположное неравенство:

$$
\mathcal{I}\left(s, t_{*}\right)<0 \text {. }
$$

Покажем, что тогда $t_{*}$ можно увеличить.

1) Покажем, что можно считать, что выполняется неравенство

$$
\phi\left(p_{0}+p_{*}-\chi_{2}\left(s_{0}\right)\right) \leqslant \phi\left(\chi_{1}\left(t_{*}\right)\right) .
$$


Действительно, так как $\mathcal{I}\left(s, t_{*}\right)$ - непрерывная функция от $s, \mathcal{I}\left(\widetilde{a}, t_{*}\right)>0$ в силу определения точек $p_{0}, p_{1}, p_{*}$, график $\phi(p)$ обязан пересечь прямую $z=\phi\left(\chi_{1}\left(t_{*}\right)\right)$ на промежутке $\left(s_{0}, \widetilde{a}\right)$.

Пусть $s_{1}=\min \left\{s \in\left(s_{0}, \widetilde{a}\right) \mid \phi\left(p_{0}+p_{*}-\chi_{2}(s)\right)=\phi\left(\chi_{1}\left(t_{*}\right)\right)\right\}$. Тогда для всех $s \in\left[s_{0}, s_{1}\right)$ верно неравенство $\phi\left(p_{0}+p_{*}-\chi_{2}(s)\right)>\phi\left(\chi_{1}\left(t_{*}\right)\right)$. Заметим теперь, чTо

$$
\begin{gathered}
0>\mathcal{I}\left(s_{0}, t_{*}\right)=\int_{\chi_{1}\left(t_{*}\right)}^{p_{0}+p_{*}-\chi_{2}\left(s_{0}\right)}\left(\phi(\tau)-\phi\left(\chi_{1}\left(t_{*}\right)\right)\right) d \tau= \\
=\int_{\chi_{1}\left(t_{*}\right)}^{p_{0}+p_{*}-\chi_{2}\left(s_{1}\right)}\left(\phi(\tau)-\phi\left(\chi_{1}\left(t_{*}\right)\right)\right) d \tau+ \\
\quad+\int_{p_{0}+p_{1}-\chi_{2}\left(s_{1}\right)}^{p_{0}+p_{*}-\chi_{2}\left(s_{0}\right)}\left(\phi(\tau)-\phi\left(\chi_{1}\left(t_{*}\right)\right)\right) d \tau>\mathcal{I}\left(s_{1}, t_{*}\right),
\end{gathered}
$$

т.е. точка $s_{1}$ удовлетворяет неравенству $\mathcal{I}\left(s_{1}, t_{*}\right)<0$, и, выбрав ее в качестве $s_{0}$, получим, что неравенство (10) также выполнено.

2) Покажем, что если найдется $s \in\left(s_{0}, \widetilde{a}\right]$ такое, что

$$
\mathcal{I}\left(s, t_{*}\right)<0, \quad \phi\left(p_{0}+p_{*}-\chi_{2}(s)\right)=\phi\left(\chi_{1}\left(t_{*}\right)\right),
$$

то $t_{*}$ можно увеличить.

Действительно, из условий на $\chi_{j}$ следует, что

$$
\phi\left(p_{0}+p_{*}-\chi_{2}(s)\right)=\phi\left(\chi_{1}\left(t_{*}\right)\right)=\phi\left(\chi_{1}(s)\right)
$$

откуда, в свою очередь, имеем, что $\mathcal{I}\left(s, t_{*}\right)=\mathcal{I}(s, s)<0$. Но так как при $t$, достаточно близких к $\widetilde{a}$, выполняется $\mathcal{I}(t, t)>0$, в силу непрерывности $\mathcal{I}(t, t)$ найдется $t_{1}>s>t_{*}$ такое, что $\mathcal{I}\left(t_{1}, t_{1}\right)=0$, т.е. $t_{*}$ можно увеличить.

3) Пусть $s_{1}, s_{2}$ таковы, что для всех $s \in\left(s_{1}, s_{2}\right)$ имеет место (11). Тогда

$$
\begin{aligned}
\mathcal{I}\left(s_{1}, t_{*}\right)=\int_{\chi_{1}\left(t_{*}\right)}^{p_{0}+p_{*}-\chi_{2}\left(s_{1}\right)}\left(\phi(\tau)-\phi\left(\chi_{1}\left(t_{*}\right)\right)\right) d \tau= & \left(\phi(\tau)-\phi\left(\chi_{1}\left(t_{*}\right)\right)\right) d \tau+ \\
=\int_{\chi_{1}\left(t_{*}\right)}^{p_{0}+p_{*}-\chi_{2}(s)} & \quad+\int_{p_{0}+p_{*}-\chi_{2}(s)}^{p_{0}+p_{*}-\chi_{2}\left(s_{1}\right)}\left(\phi(\tau)-\phi\left(\chi_{1}\left(t_{*}\right)\right)\right) d \tau<\mathcal{I}\left(s, t_{*}\right),
\end{aligned}
$$

и, более того, аналогичным образом можно показать, что $\mathcal{I}\left(s, t_{*}\right)$ строго монотонно возрастает по $s$ на интервале $\left(s_{1}, s_{2}\right)$.

Следовательно, функция $\mathcal{I}\left(s, t_{*}\right)$ переменной $s$ не может более одного раза менять знак на промежутке $\left(s_{1}, s_{2}\right)$ таком, что для всех $s \in\left(s_{1}, s_{2}\right)$ верно неравенство $\phi\left(p_{0}+p_{*}-\chi_{2}(s)\right)<\phi\left(\chi_{1}\left(t_{*}\right)\right)$. Но отсюда и из двух предыдущих шагов следует, что при указанном в начале доказательства предположении $t_{*}$ можно увеличить, что противоречит тому, что по условию $t_{*}$ максимально. Лемма 5 доказана. 
ЛЕММА 6 (о КОРРЕКТНОСТИ КРАТЧАЙШЕГО ПУТИ ВПРАВО). Пустъ $\phi \in \mathcal{B}$ удовлетворяет условиям леммы 4. Пусть также $p_{1}, p_{*}, p_{0}, p_{2}-$ точки, описанные в доказательстве леммы 4, функиии $h_{j}(t) \in C([0, a])$ таковы, что

$$
\begin{gathered}
h_{1}(0)=p_{1}, \quad h_{1}(a)=p_{*}, \quad h_{2}(0)=p_{0}, \quad h_{2}(a)=p_{2}, \\
\forall t \in[0, a]: \phi\left(h_{1}(t)\right)=\phi\left(h_{2}(t)\right),
\end{gathered}
$$

$h_{2}(t)$ - монотонно возрастающая функция.

Обозначим

$$
\mathcal{J}(s, t)=\int_{h_{1}(t)}^{h_{2}(s)}\left(\phi(\tau)-\phi\left(h_{1}(t)\right)\right) d \tau .
$$

Пусть также $t_{*} \in[0, a]$ такое, что

$$
\mathcal{J}\left(t_{*}, t_{*}\right)=0, \quad r\left(t_{*}\right)=\min \left\{t \in\left[0, t_{*}\right] \mid \phi\left(h_{1}\left(t_{*}\right)\right)=\phi\left(h_{2}(t)\right)\right\} .
$$

Тогда, если существует $s_{0} \in\left[r\left(t_{*}\right), t_{*}\right]$ такое, что $\mathcal{J}\left(s_{0}, t_{*}\right)<0$, то найдется $t^{*} \in\left[0, t_{*}\right)$ maкое, что $\mathcal{J}\left(t^{*}, t^{*}\right)=0$.

До ка з а тель ст в о. Схема доказательства полностью аналогична доказательству предыдущей леммы.

1) Можно считать, что $\phi\left(h_{2}\left(s_{0}\right)\right) \leqslant \phi\left(h_{1}\left(t_{*}\right)\right)$.

Действительно, если имеет место противоположное неравенство, то, т.к. $s_{0} \geqslant r\left(t_{*}\right)$, найдется $s_{1} \in\left(r\left(t_{*}\right), s_{0}\right)$ такое, что $\phi\left(h_{2}\left(s_{1}\right)\right)=\phi\left(h_{1}\left(t_{*}\right)\right)$, причем для всех $s \in\left(s_{1}, s_{0}\right)$ имеет место неравенство $\phi\left(h_{2}(s)\right)>\phi\left(h_{1}\left(t_{*}\right)\right)$. Но тогда

$$
\begin{aligned}
0>\mathcal{J}\left(s_{0}, t_{*}\right)=\int_{h_{1}\left(t_{*}\right)}^{h_{2}\left(s_{0}\right)}\left(\phi(\tau)-\phi\left(h_{1}\left(t_{*}\right)\right)\right) d \tau= & \\
=\int_{h_{1}\left(t_{*}\right)}^{h_{2}\left(s_{1}\right)}\left(\phi(\tau)-\phi\left(h_{1}\left(t_{*}\right)\right)\right) d \tau+ & \\
& +\int_{h_{2}\left(s_{1}\right)}^{h_{2}\left(s_{0}\right)}\left(\phi(\tau)-\phi\left(h_{1}\left(t_{*}\right)\right)\right) d \tau>\mathcal{J}\left(s_{1}, t_{*}\right),
\end{aligned}
$$

и можно рассматривать $s_{1}$ вместо $s_{0}$.

2) Заметим, что

$$
\mathcal{J}(0,0)=\int_{p_{1}}^{p_{0}}\left(\phi(\tau)-\phi\left(p_{0}\right)\right) d \tau>0
$$

так как $p_{0}$ - точка минимума $\phi(p)$. Отсюда, если для некоторого $s \in\left[0, s_{0}\right]$ верно неравенство $\mathcal{J}\left(s, t_{*}\right)<0$ и при этом $\phi\left(h_{2}(s)\right)=\phi\left(h_{1}\left(t_{*}\right)\right)$, то $h_{1}(s)=$ $=h_{1}\left(t_{*}\right)$, а значит, $\mathcal{J}(s, s)=\mathcal{J}\left(s, t_{*}\right)<0$, т.е. $t_{*}$ можно уменьшить в силу непрерывности $\mathcal{J}$.

3) Пусть $s_{1}, s_{2}$ таковы, что для всех $s \in\left(s_{1}, s_{2}\right)$ имеют место неравенства $\mathcal{J}\left(s, t_{*}\right)<0$ и $\phi\left(h_{2}(s)\right)<\phi\left(h_{1}\left(t_{*}\right)\right)$. Тогда 


$$
\begin{aligned}
& \mathcal{J}\left(s_{1}, t_{*}\right)=\int_{h_{1}\left(t_{*}\right)}^{h_{2}\left(s_{1}\right)}\left(\phi(\tau)-\phi\left(h_{1}\left(t_{*}\right)\right)\right) d \tau= \\
& \quad=\mathcal{J}\left(s_{2}, t_{*}\right)-\int_{h_{2}\left(s_{1}\right)}^{h_{2}\left(s_{2}\right)}\left(\phi(\tau)-\phi\left(h_{1}\left(t_{*}\right)\right)\right) d \tau>J\left(s_{2}, t_{*}\right),
\end{aligned}
$$

и аналогичным образом можно показать строгое монотонное убывание по $s$ функции $\mathcal{J}\left(s, t_{*}\right)$ на $\left(s_{1}, s_{2}\right)$. Следовательно, $\mathcal{J}\left(s, t_{*}\right)$ не может более одного раза менять знак на $\left(s_{1}, s_{2}\right)$. Отсюда и из предыдущих шагов следует утверждение леммы. Лемма 6 доказана.

Перейдем теперь к доказательству существования локального выравнивания, удовлетворяющего условию корректности.

ЛЕмма 7 (ОБ АЛЬТЕРнатИвЕ). Пусть $\phi \in \mathcal{B}$ удовлетворяет условиям леммы 4. Пусть также $p_{1}, p_{*}, p_{0}, p_{2}$ - точки, описанные в доказательстве леммъь 4, бункиии $h_{j}(t) \in C([0, a])$ таковь, что

$$
\begin{gathered}
h_{1}(0)=p_{1}, \quad h_{1}(a)=p_{*}, \quad h_{2}(a)=p_{0}, \quad h_{2}(a)=p_{2}, \\
\forall t \in[0, a]: \phi\left(h_{1}(t)\right)=\phi\left(h_{2}(t)\right),
\end{gathered}
$$

$h_{2}(t)$ - монотонно возрастающая функиия. Пусть такэсе

$$
t_{*}=\min \{t \in[0, a] \mid \mathcal{J}(t, t)=0\} .
$$

Тогда верно одно из двух утверждении:

(а) для всех $p \in\left[h_{1}\left(t_{*}\right), h_{2}\left(t_{*}\right)\right]$ верно неравенство

$$
\int_{h_{1}\left(t_{*}\right)}^{p} \phi(\tau) d \tau \geqslant \phi\left(h_{1}\left(t_{*}\right)\right)\left(p-h_{1}\left(t_{*}\right)\right),
$$

т.е. локальное выравнивание $\mathcal{E}_{\left[h_{1}\left(t_{*}\right), h_{2}\left(t_{*}\right)\right]}$ удовлетворяет условию корректности (определение 6);

(б) найдутся $\alpha, \beta \in\left[p_{1}, p_{0}\right)$ такие, что $\mathcal{E}_{[\alpha, \beta]}$ применимо к $\phi$ и удовлетворяет условию корректности.

Доказательство. Пусть утверждение (а) не выполнено. Докажем, что тогда выполнено (б). Действительно, т.к. (а) не выполнено, то по лемме 6 найдется $p_{c} \in\left[h_{1}\left(t_{*}\right), r\left(t_{*}\right)\right)$ такое, что

$$
\mathcal{H}\left(p_{c}\right)=\int_{h_{1}\left(t_{*}\right)}^{p_{c}}\left(\phi(\tau)-\phi\left(h_{1}\left(t_{*}\right)\right)\right) d \tau<0 .
$$

Пусть $\bar{p}=\max \left\{p \in\left(p_{*}, p_{0}\right) \mid \phi(p)=\phi\left(h_{1}\left(t_{*}\right)\right)\right\}$. Заметим, что для всех $p \in\left(\bar{p}, r\left(t_{*}\right)\right)$ имеет место неравенство

$$
\mathcal{H}(p)=\mathcal{H}(\bar{p})+\int_{\bar{p}}^{p}\left(\phi(\tau)-\phi\left(h_{1}\left(t_{*}\right)\right)\right) d \tau<\mathcal{H}(\bar{p}),
$$

и в силу леммы 6 верно неравенство $\mathcal{H}\left(r\left(t_{*}\right)\right)>0$. Отсюда получаем, что для всех $p \in\left(\bar{p}, r\left(t_{*}\right)\right)$ имеет место неравенство $\mathcal{H}(p)>0$, и в силу непрерывности $\mathcal{H}(p)$ из неравенств $\mathcal{H}\left(p_{c}\right)<0$ и $h_{1}\left(t_{*}\right) \leqslant p_{c}<r\left(t_{*}\right)$ следует неравенство $p_{c}<\bar{p}$. 
Заметим теперь, что можно считать, что

$$
\phi\left(p_{c}\right) \geqslant \phi\left(h_{1}\left(t_{*}\right)\right)
$$

Действительно, если выполнено противоположное неравенство, то положим

$$
p_{R}=\min \left\{p \in\left(p_{c}, \bar{p}\right) \mid \phi(p)=\phi\left(h_{1}\left(t_{*}\right)\right)\right\}
$$

Заметим, что в этом случае

$$
\begin{aligned}
\mathcal{H}\left(p_{R}\right)=\int_{h_{1}\left(t_{*}\right)}^{p_{R}}\left(\phi(\tau)-\phi\left(h_{1}\left(t_{*}\right)\right)\right) d \tau & = \\
=\int_{h_{1}\left(t_{*}\right)}^{p_{c}}\left(\phi(\tau)-\phi\left(h_{1}\left(t_{*}\right)\right)\right) d \tau+ & \\
& +\int_{p_{c}}^{p_{R}}\left(\phi(\tau)-\phi\left(h_{1}\left(t_{*}\right)\right)\right) d \tau<\mathcal{H}\left(p_{c}\right)<0,
\end{aligned}
$$

так как второй интеграл всегда отрицателен. Но это означает, что для $p_{R}$ также верно $\mathcal{H}\left(p_{R}\right)<0$, и, взяв $p_{R}$ вместо $p_{c}$, получаем требуемое неравенство (12).

Далее применим обобщенную лемму о непрерывном обходе (лемма 2) к паре функций $f(p)=\left.\phi\right|_{\left[p_{1}, p_{*}\right]}, g(p)=\phi\left(p_{*}+p_{0}-p\right), p \in\left[p_{*}, p_{0}\right]$. Получим пару функций $\chi_{j}(t) \in C([0, \widetilde{a}])$ таких, что $\chi_{2}$ монотонно возрастает, и $f\left(\chi_{1}(t)\right)=g\left(\chi_{2}(t)\right)$. Но тогда найдутся $t_{0} \in[0, \widetilde{a}], s \in[0, \widetilde{a}]$ такие, что

$$
h_{1}\left(t_{*}\right)=\chi_{1}\left(t_{0}\right), \quad \bar{p}=p_{0}+p_{*}-\chi_{2}\left(t_{0}\right), \quad p_{c}=p_{0}+p_{*}-\chi_{2}(s) .
$$

Из изложенного выше получаем $\mathcal{I}\left(t_{0}, t_{0}\right) \geqslant 0, \mathcal{I}\left(s, t_{0}\right)<0$. Но тогда в силу строго монотонного возрастания $\phi(p)$ на промежутке $\left(p_{1}, p_{*}\right)$ и неравенства $(12)$ получаем, что $\chi_{1}(s)>\chi_{1}\left(t_{0}\right)$, и далее

$$
\begin{aligned}
& \mathcal{I}(s, s)=\int_{\chi_{1}(s)}^{p_{*}+p_{0}-\chi_{2}(s)}\left(\phi(\tau)-\phi\left(\chi_{1}(s)\right) d \tau=\right. \\
& =\int_{\chi_{1}\left(t_{0}\right)}^{p_{*}+p_{0}-\chi_{2}(s)}\left(\phi(\tau)-\phi\left(\chi_{1}\left(t_{0}\right)\right) d \tau+\int_{\chi_{1}(s)}^{p_{*}+p_{0}-\chi_{2}(s)}\left(\phi\left(\chi_{1}\left(t_{0}\right)\right)-\phi\left(\chi_{1}(s)\right) d \tau-\right.\right. \\
& -\int_{\chi_{1}\left(t_{0}\right)}^{\chi_{1}(s)}\left(\phi(\tau)-\phi\left(\chi_{1}\left(t_{0}\right)\right)\right) d \tau<\mathcal{I}\left(s, t_{0}\right)<0 .
\end{aligned}
$$

Но тогда из неравенств $\mathcal{I}(s, s)<0, \mathcal{I}\left(t_{0}, t_{0}\right) \geqslant 0$ в силу непрерывности $\mathcal{I}$ следует существование $t_{1} \in\left[t_{0}, s\right)$ такого, что $\mathcal{I}\left(t_{1}, t_{1}\right)=0$. Следовательно, можно воспользоваться леммой о корректности кратчайшего пути влево (лемма 5 ) и получить утверждение (б) для

$$
t_{2}=\max \{t \in[0, \widetilde{a}) \mid \mathcal{I}(t, t)=0\}, \quad \alpha=\chi_{1}\left(t_{2}\right), \quad \beta=p_{*}+p_{0}-\chi_{2}\left(t_{2}\right) .
$$

Лемма 7 доказана. 
ЗАмЕчАниЕ 5. Из леммы об альтернативе при помощи рассуждений, аналогичных рассуждениям замечания 2 , получаем, что для $\phi \in \mathcal{B}$ существует полное выравнивание.

\section{7. Единственность полного выравнивания.}

Лемма 8. Пусть $\phi \in \mathcal{B}$. Тогда $\mathcal{E}_{*} \phi$ единственно.

Д о каз а тель ст в о. Предположим противное. Пусть $\psi_{1}(p), \psi_{2}(p)$ - два различных полных выравнивания. Обозначим $\left\{\left[p_{k}^{(j)}, q_{k}^{(j)}\right]\right\}_{k=1}^{m_{j}}, j=1,2$, набор отрезков $I_{k}^{(j)}$ из определения полного выравнивания для $\psi_{j}$. Без ограничения общности можно считать, что вне отрезков $I_{k}^{(j)}$ функции $\psi_{j}$ строго монотонны. Пусть $p_{1}^{(1)}<p_{1}^{(2)}$. Заметим, что отрезки $\left[p_{1}^{(1)}, q_{1}^{(1)}\right]$ и $\left[p_{1}^{(2)}, q_{1}^{(2)}\right]$ обязаны пересекаться - иначе $\phi\left(p_{1}^{(1)}\right)=\phi\left(q_{1}^{(1)}\right)$, что противоречит определению отрезков $I_{k}^{(j)}$. Тогда возможны три случая:

(i) $q_{1}^{(1)}<q_{1}^{(2)}$

(ii) $q_{1}^{(1)}=q_{1}^{(2)}$,

(iii) $q_{1}^{(1)}>q_{1}^{(2)}$.

В случае (i):

$$
\int_{0}^{q_{1}^{(1)}} \phi(p) d p=\int_{0}^{q_{1}^{(1)}} \psi_{1}(p) d p \geqslant \int_{0}^{q_{1}^{(1)}} \psi_{2}(p) d p
$$

но, так как $\psi(p)$ монотонно не убывает на $\left[p_{1}^{(1)}, p_{1}^{(2)}\right]$ (иначе $\psi_{2}$ - немонотонная, а значит, не является полным выравниванием), имеем

$$
\int_{p_{1}^{(1)}}^{p_{1}^{(2)}} \psi_{2}(p) d p=\int_{p_{1}^{(1)}}^{p_{1}^{(2)}} \psi(p) d p>\int_{p_{1}^{(1)}}^{p_{1}^{(2)}} \psi_{1}(p) d p
$$

а также

$$
\int_{p_{1}^{(2)}}^{q_{1}^{(1)}} \psi_{2}(p) d p=\int_{p_{1}^{(2)}}^{q_{1}^{(1)}} \phi\left(p_{1}^{(2)}\right) d p>\int_{p_{1}^{(2)}}^{q_{1}^{(1)}} \phi\left(p_{1}^{(1)}\right) d p=\int_{p_{1}^{(2)}}^{q_{1}^{(1)}} \psi_{1}(p) d p
$$

Но из (14), (15) следует

$$
\int_{0}^{q_{1}^{(1)}} \psi_{2}(p) d p>\int_{0}^{q_{1}^{(1)}} \psi_{1}(p) d p
$$

что противоречит равенству (13). Следовательно, случай (i) невозможен.

Невозможность случая (ii) доказывается полностью аналогично.

Пусть имеет место случай (iii). Но тогда на интервале $\left(q_{1}^{(2)}, q_{1}^{(1)}\right)$ функция $\psi_{2}(p)$ монотонно не убывает, следовательно,

$$
\int_{q_{1}^{(2)}}^{q_{1}^{(1)}} \psi_{2}(p) d p \geqslant \int_{q_{1}^{(2)}}^{q_{1}^{(1)}} \psi_{2}\left(q_{1}^{(2)}\right) d p>\int_{q_{1}^{(2)}}^{q_{1}^{(1)}} \psi_{1}\left(q_{1}^{(2)}\right) d p
$$


откуда, в свою очередь, следует неравенство

$$
\int_{0}^{q_{1}^{(1)}} \psi_{2}(p) d p>\int_{0}^{q_{1}^{(1)}} \psi_{1}(p) d p=\int_{0}^{q_{1}^{(1)}} \phi(p) d p
$$

что противоречит условию корректности для $\psi_{2}$. Таким образом, случай (iii) также невозможен.

Так как никакой из случаев (i)-(iii) не может реализоваться, то предположение о том, что $p_{1}^{(1)}<p_{1}^{(2)}$, неверно. В силу симметрии между $\psi_{1}$ и $\psi_{2}$ не может быть выполнено и неравенство $p_{1}^{(1)}>p_{1}^{(2)}$. Следовательно, $p_{1}^{(1)}=p_{1}^{(2)}$.

Аналогично доказывается, что $q_{1}^{(1)}=q_{1}^{(2)}$ и т. д., что и означает равенство $\psi_{1}=\psi_{2}$, т. е. единственность полного выравнивания. Лемма 8 доказана.

ЗАмечание 6. Существование полного выравнивания для немонотонной $\psi \in \mathcal{B}$ следует из замечания 5. Для монотонной $\psi$ можно положить $\mathcal{E}_{*} \psi=\psi$. Таким образом, теорема о корректности полного выравнивания доказана.

\section{8. Полное выравнивание и аффинные преобразования независи-} мой переменной. Расширим определение полного выравнивания на несколько простых случаев.

ОПРедЕЛЕние 7 . Пусть $\phi(p)$ такова, что $\phi(1-p) \in \mathcal{B}$. Обозначим $\psi(p)=$ $=\phi(1-p), \widetilde{\psi}(p)=\mathcal{E}_{*} \psi(p)$. Полным выравниванием для исходной функции $\phi(p)$ назовем $\mathcal{E}_{*} \phi(p)=\widetilde{\psi}(1-p)$.

Корректность этого определения очевидно следует из теоремы о корректности полного выравнивания (теорема 1) для функций из $\mathcal{B}$. Обобщением этого определения на случай произвольной аффинной замены независимой переменной является следующее определение.

ОПРЕДЕЛЕниЕ 8. Пусть $\phi(p)$ такова, что для некоторых $\alpha \in(\mathbb{R} \backslash\{0\})$, $\beta \in \mathbb{R}$ функция $\psi(q)=\phi(\alpha q+\beta) \in \mathcal{B}$. Полным выравниванием для исходной функции $\phi(p)$ назовем $\mathcal{E}_{*} \phi(p)=\left(\mathcal{E}_{*} \psi\right)\left(\frac{p-\beta}{\alpha}\right)$.

Корректность этого определения также следует из теоремы 1. Заметим также, что требование 2) в определении класса $\mathcal{B}$ можно ослабить и при этом по-прежнему корректно определить полное выравнивание.

ОПРедЕлЕНиЕ 9. Будем обозначать $\Upsilon$ класс функций $\phi(p)$ таких, что:

1) $\phi(p) \in C([0,1])$;

2) $\forall p \in(0,1): \phi(0) \leqslant \phi(p) \leqslant \phi(1)$;

$3)$ отрезок $[0,1]$ можно представить в виде конечного объединения отрезков:

$$
[0,1]=\bigcup_{j=1}^{k}\left[m_{j-1}, m_{j}\right]
$$

так, что $m_{0}<m_{1}<\cdots<m_{k}$, и на каждом из отрезков $\left[m_{j-1}, m_{j}\right]$ функция $\phi(p)$ либо строго монотонна, либо постоянна.

ОпредЕЛЕНиЕ 10. Пусть $\phi(p) \in \Upsilon$. Возьмем $\varepsilon>0$ и определим функцию 
$\phi_{\varepsilon}(p)$ равенством

$$
\phi_{\varepsilon}(p)=\left\{\begin{array}{cc}
\phi(0)+p, & -\varepsilon \leqslant p \leqslant 0 \\
\phi(p), & 0 \leqslant p \leqslant 1 \\
\phi(1)+p, & 1 \leqslant p \leqslant 1+\varepsilon
\end{array}\right.
$$

Тогда нетрудно видеть, что $\phi_{\varepsilon}(p)$ допускает полное выравнивание согласно определению 8. Полным выравниванием для исходной функции $\phi(p)$ назовем ограничение функции $\mathcal{E}_{*} \phi_{\varepsilon}(p)$ на отрезок $[0,1]$.

Нетрудно видеть, что определение 10 корректно и допускает обобщение, аналогичное определению 8.

\section{9. Связъ условия корректности с условием допустимого раз-} рыва. Пусть теперь $\gamma \in \mathcal{K}_{R}, S_{t} \gamma$ - соответствующее геометрическое решение. Предположим пока для простоты, что существует ровно один отрезок $\left[x_{1}, x_{2}\right]$ такой, что $S_{t} \gamma$ не является графиком функции $F_{\gamma}(x)$ на этом отрезке, $S_{t} \gamma$ является на этом отрезке графиком функции $\phi(p)$ при $p \in\left[p_{1}, p_{2}\right]$, этот отрезок - максимальное связное множество, удовлетворяющее этим условиям, и $\phi(p)$ удовлетворяет двойному неравенству $\phi\left(p_{1}\right) \leqslant \phi(p) \leqslant \phi\left(p_{2}\right)$ для всех $p \in\left[p_{1}, p_{2}\right]$. Тогда, согласно рассуждениям предыдущего пункта, для функции $\phi(p)$ определено полное выравнивание $\mathcal{E}_{*} \phi(p)$.

Обозначим $\mathcal{E}_{*} S_{t} \gamma$ кривую, являющуюся объединением графика $\mathcal{E}_{*} \phi(p)$ и ограничения $S_{t} \gamma$ на множество $\left(-\infty, x_{1}\right) \cup\left(x_{2},+\infty\right)$. Тогда существует кусочнонепрерывная ограниченная функция $F_{\gamma}(x)$ и множество $A_{\gamma}$ меры нуль такие, что $\mathcal{E}_{*} S_{t} \gamma \cap\left\{x \notin A_{\gamma}\right\}$ - график функции $F_{\gamma}(x)$.

Пусть $x_{0}$ - точка разрыва $F_{\gamma}(x)$. Тогда $x_{0} \in A_{\gamma}$, и, обозначая

$$
p_{L}=F_{\gamma}\left(x_{0}-0\right), \quad p_{R}=F_{\gamma}\left(x_{0}+0\right),
$$

получаем, что $\mathcal{E}_{*} \phi\left(p_{L}\right)=\mathcal{E}_{*} \phi\left(p_{R}\right)$.

В силу неравенства $\phi\left(p_{1}\right) \leqslant \phi(p) \leqslant \phi\left(p_{2}\right)$ имеем $\phi(p) \in \Upsilon$, и из определения 10 следует, что $\mathcal{E}_{*} \phi(p)$ монотонно неубывающая, откуда $p_{L}<p_{R}$. Из условия корректности (определение 6) для всех $p \in\left[p_{L}, p_{R}\right]$ имеет место неравенство

$$
\int_{p_{L}}^{p} \phi(\tau) d \tau \geqslant \int_{p_{L}}^{p}\left(\mathcal{E}_{*} \phi\right)(\tau) d \tau .
$$

Заметим, что все приведенные выше рассуждения можно проделать вне зависимости от того, зависит ли гамильтониан от переменной $x$. Рассмотрим теперь частный случай, когда гамильтониан от $x$ не зависит и имеет вид $\Phi(p)$. Тогда, задав начальную кривую $\gamma$ в параметрическом виде

$$
\gamma=\left\{(x, p) \mid x=X_{\gamma}(\xi), p=P_{\gamma}(\xi), \xi \in \mathbb{R}\right\}
$$

получаем, что

$$
S_{t} \gamma=\left\{\left(X_{\gamma}(\xi)+t \Phi^{\prime}\left(P_{\gamma}(\xi)\right), P_{\gamma}(\xi)\right) \mid \xi \in \mathbb{R}\right\}
$$

Обозначим $\xi_{L}$ и $\xi_{R}$ точки такие, что $P_{\gamma}\left(\xi_{L}\right)=p_{L}, P_{\gamma}\left(\xi_{R}\right)=p_{R}$. Тогда точкам $p \in\left[p_{L}, p_{R}\right]$ соответствуют значения параметра $\xi \in\left[\xi_{L}, \xi_{R}\right]$. После этого, преобразуя (16), получаем 


$$
\begin{aligned}
0 \leqslant \int_{p_{L}}^{p}\left(\phi(\tau)-x_{0}\right) d \tau & =\int_{\xi_{L}}^{\xi}\left(X(\eta)+t \Phi^{\prime}\left(P_{\gamma}(\eta)\right)-x_{0}\right) P^{\prime}(\eta) d \eta= \\
& =\int_{\xi_{L}}^{\xi}\left(X_{\gamma}(\eta)-x_{0}\right) P^{\prime}(\eta) d \eta+t \Phi\left(P_{\gamma}(\xi)\right)-t \Phi\left(P_{\gamma}\left(\xi_{L}\right)\right) .
\end{aligned}
$$

Заметим теперь, что $x_{0}=\phi\left(p_{L}\right)=X_{\gamma}\left(\xi_{L}\right)+t \Phi^{\prime}\left(p_{L}\right)$. Кроме того, для того чтобы описанная ситуация имела место для достаточно малых $t$, необходимо, чтобы $\gamma$ была графиком неубывающей функции $p=P_{\gamma}\left(X_{\gamma}^{-1}(x)\right)$. Но тогда, продолжая преобразования, получаем

$$
\begin{aligned}
\int_{\xi_{L}}^{\xi}\left(X_{\gamma}(\eta)-X_{\gamma}\left(\xi_{L}\right)\right) P^{\prime}(\eta) d \eta+ & \\
& +t\left(\Phi\left(P_{\gamma}(\xi)\right)-\Phi\left(P_{\gamma}\left(\xi_{L}\right)\right)-\Phi^{\prime}\left(p_{L}\right)\left(P_{\gamma}(\xi)-P_{\gamma}\left(\xi_{L}\right)\right)\right) \geqslant 0
\end{aligned}
$$

В силу указанного свойства начальной кривой $\gamma$ интегральное слагаемое в этом неравенстве неотрицательно, но может обратиться в нуль, если $\gamma-$ ломаная, содержащая вертикальный отрезок. В этом случае получаем

$$
\Phi\left(P_{\gamma}(\xi)\right)-\Phi\left(P_{\gamma}\left(\xi_{L}\right)\right)-\Phi^{\prime}\left(p_{L}\right)\left(P_{\gamma}(\xi)-P_{\gamma}\left(\xi_{L}\right)\right) \geqslant 0
$$

Осталось заметить, что последнее неравенство совпадает с предложенным Н. Введенской [15] условием допустимости разрыва-хорда, соединяющая точки $p_{L}$ и $p_{R}$, не пересекает график $\Phi(p)$. Таким образом, функция $F_{\gamma}(x)$ в каждой из точек разрыва удовлетворяет условию допустимости разрыва.

\subsection{0. Обобщение полного выравнивания на случай несложннх кривъц.}

ОПРедЕЛЕНие 11. Кривую $\gamma \in \mathcal{K}_{R}$ будем называть несложной, если найдется конечное множество отрезков $I_{1}, \ldots, I_{N}$ таких, что:

1) для всех $k=1, \ldots, N$ множество $\gamma \cap\left\{x \in I_{k}\right\}$ - график непрерывной функции, зависящей от $p$;

2) множество $\gamma \cap\left(\mathbb{R} \backslash\left(\cup_{k=1}^{N} I_{k}\right)\right.$ - график кусочно-непрерывной функции, зависящей от $x$.

Будем далее считать, что $\gamma$ параметризована непрерывным образом при помощи параметра $\xi$, т.е.

$$
\gamma=\left\{(x, p) \mid x=X_{\gamma}(\xi), p=P_{\gamma}(\xi), \xi \in \mathbb{R}\right\}
$$

причем для некоторого $R_{1}>0$ при $\xi<-R_{1}$ имеют место соотношения $X_{\gamma}(\xi)<-R, P_{\gamma}(\xi)=C_{-}(\gamma)$. Множество всех несложных кривых с такой параметризацией будем обозначать $\widehat{\mathcal{K}}_{R}$. Заметим, что в силу конечности скорости распространения возмущений, если $\gamma \in \widehat{\mathcal{K}}_{R}$, то для всех достаточно малых $t>0: S_{t} \gamma \in \widehat{\mathcal{K}}_{R}$.

Определим полное выравнивание для несложной кривой. Для каждого $k \in\{1, \ldots, N\}$ положим $J_{k}=\left[\xi_{L}^{(k)}, \xi_{R}^{(k)}\right]$ так, чтобы $X_{\gamma}\left(J_{k}\right)=I_{k}$. Пусть также $\phi_{k}(p)$ - функция, график которой на $\left[P_{\gamma}\left(\xi_{L}^{(k)}\right), P_{\gamma}\left(\xi_{R}^{(k)}\right)\right]$ совпадает с $\gamma \cap\left\{x \in I_{k}\right\}$. 
ОПредЕлЕниЕ 12. Полным выравниванием для $\gamma \in \widehat{\mathcal{K}}_{R}$ будем называть непрерывную кривую (далее будем обозначать ее $\mathcal{E}_{*} \gamma$ ), которая строится по следующему правилу: кривая $\mathcal{E}_{*} \gamma$ является объединением участков кривой $\gamma$ и отрезков, параллельных оси $O p$. При этом каждый такой отрезок $\left\{\left(x^{*}, p\right) \mid\right.$ $\left.p \in\left[p_{1}^{*}, p_{2}^{*}\right]\right\}$ проектируется на $I_{k}$ для некоторого $k$, причем существуют $\xi_{1}^{*}, \xi_{2}^{*} \in$ $J_{k}$ такие, что:

1) $P_{\gamma}\left(\xi_{1}^{*}\right)=p_{1}^{*}, P_{\gamma}\left(\xi_{2}^{*}\right)=p_{2}^{*}$

2) $\int_{\left(x^{*}, p_{1}^{*}\right)}^{\left(x^{*}, p_{2}^{*}\right)} X_{\gamma} d P_{\gamma}=x^{*}\left(p_{2}^{*}-p_{1}^{*}\right)$;

3) для всех $\xi$, лежащих между $\xi_{1}^{*}$ и $\xi_{2}^{*}$, имеет место неравенство

$$
\int_{\left(x^{*}, p_{1}^{*}\right)}^{\left(X_{\gamma}(\xi), P_{\gamma}(\xi)\right)}\left(X_{\gamma}-x^{*}\right) d P_{\gamma} \geqslant 0 .
$$

Нетрудно видеть, что приведенное выше определение корректно, является обобщением определения выравнивания, подробно рассматривавшегося выше, и неравенство (17) согласовано с условием допустимого разрыва в обсуждавшемся в предыдущем пункте смысле.

2.11. Связъ полного выравнивания несложной кривой и обобщенного решения. Пусть $\Phi(p, x) \in C^{3}$. Рассмотрим задачу Коши

$$
\left\{\begin{array}{l}
u_{t}+(\Phi(u, x))_{x}=0 \\
\left.u\right|_{t=0}=u_{0}(x)
\end{array}\right.
$$

где $u_{0}(x) \in C^{2}$ - функция, удовлетворяющая условию стабилизации.

ОПРЕДЕЛЕНиЕ 13. Будем говорить, что для функции $u_{0}(x)$ имеют место условия стабилизации, если существуют константы $u_{-}, u_{+} \in \mathbb{R}$ и $R>0$ такие, что $u_{0}(x)=u_{-}$для $x<-R, u_{0}(x)=u_{+}$для $x>R$.

Пусть $\gamma_{0}=\left\{\left(\xi, u_{0}(\xi)\right) \mid \xi \in \mathbb{R}\right\}$. Определим параметризацию траекторного решения $S_{t} \gamma_{0}$ согласованно с параметризацией начальной кривой, т. е. $(x(t, \xi), p(t, \xi))=S_{t}\left(\xi, u_{0}(\xi)\right)$. Предположим, что существует $T>0$ такое, что при $t \in[0, T]$ кривая $S_{t} \gamma_{0}$ - несложная (заметим, что это предположение выполнено для случая гамильтониана, не зависящего от $x$ ). Тогда определено выравнивание $\mathcal{E}_{*} S_{t} \gamma_{0}$. Заметим, что в силу свойств выравнивания для каждого $t \in[0, T]$ существует кусочно-непрерывная функция переменной $x-$ функция $U(t, x)$ такая, что для п.в. $x \in \mathbb{R}$ график $U(t, x)$ совпадает с $\mathcal{E}_{*} S_{t} \gamma_{0}$.

Теорема 2. Пусть для любого $c \in \mathbb{R}$ множества $\{(x, p) \mid \Phi(p, x)=c\} u$ $\left\{(x, p) \mid \frac{\partial^{2} \Phi}{\partial p^{2}}=0\right\}$ пересекаются либо по компоненте связности множества $\{(x, p) \mid \Phi(p, x)=c\}$, либо не более чем в счетном числе точек. Тогда для n.в. $t \in(0, T)$ функиия $U(t, x)$ является обобщенным решением задачи (18) в смиьсле $D^{\prime}$.

Доказ а тель ст в о. Пусть сначала $U(t, x)$ непрерывна по $x$ в некоторой окрестности точки $x_{0}$. Тогда в силу замечания 1 функция $U(t, x)-$ классическое решение. Следовательно, $U(t, x)$ - решение п.в.

Пусть теперь $U(t, x)$ разрывна при $x=x_{0}(t)$. Покажем, что $x_{0}(t) \in C^{1}$ для п.в. $t \in[0, T]$. Заметим, что существует единственное значение параметра 
$\xi_{0}(t)$ такое, что $x\left(t, \xi_{0}(t)\right)=x_{0}(t), p\left(t, \xi_{0}(t)\right)=U\left(t, x_{0}(t)-0\right)$. Аналогично обозначим $\xi_{1}(t)$ такое значение параметра, что $x\left(t, \xi_{1}(t)\right)=x_{0}(t), p\left(t, \xi_{1}(t)\right)=$ $=U\left(t, x_{0}(t)+0\right)$.

Докажем сначала, что $\xi_{0}(t), \xi_{1}(t)$ - непрерывно дифференцируемые функции для п.в. $t \in[0, T]$. Действительно, если $\xi_{j}(t)$ - интересующие нас функции, то для некоторого $a \in \mathbb{R}$ имеет место система уравнений

$$
\left\{\begin{array}{l}
x\left(t, \xi_{0}\right)=a, \\
x\left(t, \xi_{1}\right)=a, \\
\int_{\xi_{0}}^{\xi_{1}}(x(t, \xi)-a) d p(t, \xi)=0 .
\end{array}\right.
$$

Здесь $x(t, \xi)$ - решение системы ОДУ Гамильтона

$$
\begin{cases}\dot{x}=\frac{\partial \Phi}{\partial p}, & \left.x\right|_{t=0}=\xi \\ \dot{p}=-\frac{\partial \Phi}{\partial x}, & \left.p\right|_{t=0}=u_{0}(\xi)\end{cases}
$$

Так как система (20) удовлетворяет всем условиям теоремы о дифференцировании решения по параметру, $x(t, \xi)$ - непрерывно дифференцируемая функция параметра $\xi$. Пусть теперь система (19) разрешима при $t=t_{0}$ (это верно в нашем случае для $\left.a=x_{0}\left(t_{0}\right)\right)$. Тогда, вычисляя явно якобиан $J(t)$ системы (19), получаем

$$
J\left(t_{0}\right)=\left(p\left(t_{0}, \xi_{0}\left(t_{0}\right)\right)-p\left(t_{0}, \xi_{1}\left(t_{0}\right)\right) \frac{\partial x}{\partial \xi}\left(t_{0}, \xi\left(t_{0}\right)\right) \frac{\partial x}{\partial \xi}\left(t_{0}, \xi_{1}\left(t_{0}\right)\right)\right.
$$

Так как функция $U(t, x)$ разрывна при $t=t_{0}, x=x_{0}\left(t_{0}\right)$, имеем

$$
p\left(t_{0}, \xi_{0}\left(t_{0}\right)\right) \neq p\left(t_{0}, \xi_{1}\left(t_{0}\right)\right)
$$

Докажем, что два оставшихся сомножителя также не обращаются в нуль для п.в. $t_{0}$. Обозначая $q=\frac{\partial x}{\partial \xi}, r=\frac{\partial p}{\partial \xi}$ и выписывая систему уравнений в вариациях для (20), получаем

$$
\begin{cases}\dot{q}=\frac{\partial^{2} \Phi}{\partial x \partial p} q+\frac{\partial^{2} \Phi}{\partial p^{2}} r, & \left.q\right|_{t=0}=1 \\ \dot{r}=-\frac{\partial^{2} \Phi}{\partial x^{2}} q-\frac{\partial^{2} \Phi}{\partial p \partial x} r, & \left.r\right|_{t=0}=u_{0}^{\prime}(\xi)\end{cases}
$$

Обозначим для краткости $m_{11}=\frac{\partial^{2} \Phi}{\partial x \partial p}, m_{12}=\frac{\partial^{2} \Phi}{\partial p^{2}}, m_{21}=-\frac{\partial^{2} \Phi}{\partial x^{2}}$. Тогда система ОДУ (21) перепишется в виде

$$
\frac{d}{d t}\left(\begin{array}{c}
q \\
r
\end{array}\right)=\left(\begin{array}{cc}
m_{11} & m_{12} \\
m_{21} & -m_{11}
\end{array}\right)\left(\begin{array}{l}
q \\
r
\end{array}\right)
$$

Рассмотрим теперь отдельно два случая. Пусть сначала для

$$
c=\Phi\left(p\left(t_{0}, \xi_{0}\left(t_{0}\right)\right), x\left(t_{0}, \xi_{0}\left(t_{0}\right)\right)\right)
$$


множества $\{(x, p) \mid \Phi(p, x)=c\}$ и $\left\{(x, p) \mid \frac{\partial^{2} \Phi}{\partial p^{2}}=0\right\}$ пересекаются по компоненте связности множества $\{(x, p) \mid \Phi(p, x)=c\}$. Тогда, т.к. гамильтониан первый интеграл, $m_{11}=0$ для всех $t$. Но тогда для $q(t)$ имеем

$$
\dot{q}=m_{11} q,\left.\quad q\right|_{t=0}=1,
$$

т.е. $q(t)$ - решение линейного однородного ОДУ с ненулевыми начальными данными, а значит, $q(t) \neq 0$ для всех $t$, откуда следует, что $J\left(t_{0}\right) \neq 0$ для всех $t_{0} \in[0, T]$.

Пусть теперь для

$$
c=\Phi\left(p\left(t_{0}, \xi_{0}\left(t_{0}\right)\right), x\left(t_{0}, \xi_{0}\left(t_{0}\right)\right)\right)
$$

множества $\{(x, p) \mid \Phi(p, x)=c\}$ и $\left\{(x, p) \mid \frac{\partial^{2} \Phi}{\partial p^{2}}=0\right\}$ пересекаются не более чем в счетном числе точек. Тогда для всех моментов $t_{0}$ таких, что $t_{0}$ не соответствует точке пересечения, имеем $m_{12} \neq 0$.

Предположим, что $q(t)=0$ для всех $t \in\left(t_{1}, t_{2}\right)$. Тогда на этом интервале

$$
0=\dot{q}=m_{11} q+m_{12} r=m_{12} r,
$$

а значит, на этом интервале и $r(t)=0$. Но тогда в силу того, что $(21)$ - линейная однородная система ОДУ, равенство $q(t)=r(t)=0$ должно выполняться для всех $t \in \mathbb{R}$. Однако $q(0)=1 \neq 0$.

Следовательно, наше предположение неверно, и целого интервала такого, что $q(t)=0$ во всех точках интервала, не существует. Но это означает, что $J\left(t_{0}\right) \neq 0$ для п.в. $t_{0}$.

Применяя теперь теорему о системе неявных функций к (19), получаем, что $\xi_{0}(t) \in C^{1}, \xi_{1}(t) \in C^{1}$ для п.в. $t$.

Заметим теперь, что из гладкости $\xi_{0}(t)$ в силу теоремы о дифференцировании по параметру, примененной к системе $(20)$, следует, что для п. в. $t$ функция $x_{0}(t) \in C^{1}$.

Для того чтобы кусочно-гладкая $U(t, x)$ была $D^{\prime}$-решением $(18)$, необходимо и достаточно, чтобы для каждой точки разрыва $x_{0}(t)$ обнулялись коэффициенты при сингулярных членах, возникающих при прямой подстановке $U(t, x)$ в $(18)$.

Вычислим эти коэффициенты, представив $U(t, x)$ в виде

$$
U(t, x)=g(t, x)+\left(U\left(t, x_{0}(t)+0\right)-U\left(t, x_{0}(t)-0\right)\right) \theta\left(x-x_{0}(t)\right)
$$

где $g(t, x)$ - непрерывная. Тогда

$$
\begin{aligned}
\Phi(U(t, x), x)=\Psi(t, x)+\left(\Phi \left(U \left(t, x_{0}(t)\right.\right.\right. & \left.+0), x_{0}(t)\right)- \\
& \left.-\Phi\left(U\left(t, x_{0}(t)-0\right), x_{0}(t)\right)\right) \theta\left(x-x_{0}(t)\right),
\end{aligned}
$$

где $\Psi(t, x)$ непрерывна и, следовательно, равенство для сингулярной части имеет вид

$$
\dot{x_{0}}(t)\left(p\left(t, \xi_{1}(t)\right)-p\left(t, \xi_{0}(t)\right)\right)=\Phi\left(p\left(t, \xi_{1}(t)\right), x_{0}(t)\right)-\Phi\left(p\left(t, \xi_{0}(t)\right), x_{0}(t)\right) .
$$


Так как $\mathcal{E}_{*}$ - полное выравнивание, имеем

$$
\int_{\xi_{0}(t)}^{\xi_{1}(t)} x(t, \xi) d p(t, \xi)=x_{0}(t)\left(p\left(t, \xi_{1}(t)\right)-p\left(t, \xi_{0}(t)\right)\right)
$$

Дифференцируя это равенство по $t$, получаем

$$
\begin{aligned}
& \dot{\xi}_{1}(t) x\left(t, \xi_{1}(t)\right) \frac{\partial p}{\partial \xi}\left(t, \xi_{1}(t)\right)-\dot{\xi}_{0}(t) x\left(t, \xi_{0}(t)\right) \frac{\partial p}{\partial \xi}\left(t, \xi_{0}(t)\right)+ \\
& +\int_{\xi_{0}}^{\xi_{1}} \dot{x} d p(t, \xi)+\int_{\xi_{0}}^{\xi_{1}} x(t, \xi) \frac{\partial \dot{p}}{\partial \xi}(t, \xi) d \xi=\dot{x}_{0}(t)\left(p\left(t, \xi_{1}(t)\right)-p\left(t, \xi_{0}(t)\right)\right)+ \\
& \quad+x_{0}(t)\left(\frac{\partial p}{\partial \xi}\left(t, \xi_{1}(t)\right) \dot{\xi}_{1}(t)+\dot{p}\left(t, \xi_{1}(t)\right)-\frac{\partial p}{\partial \xi}\left(t, \xi_{0}(t)\right) \dot{\xi}_{0}(t)-\dot{p}\left(t, \xi_{0}(t)\right)\right) .
\end{aligned}
$$

Отсюда, приводя подобные слагаемые, получаем

$$
\begin{aligned}
& \int_{\xi_{0}}^{\xi_{1}} \dot{x} d p(t, \xi)+\int_{\xi_{0}}^{\xi_{1}} x(t, \xi) \frac{\partial \dot{p}}{\partial \xi}(t, \xi) d \xi= \\
& \quad=\dot{x}_{0}(t)\left(p\left(t, \xi_{1}(t)\right)-p\left(t, \xi_{0}(t)\right)\right)+x_{0}(t)\left(\dot{p}\left(t, \xi_{1}(t)\right)-\dot{p}\left(t, \xi_{0}(t)\right)\right) .
\end{aligned}
$$

Проинтегрируем по частям:

$$
\begin{gathered}
\int_{\xi_{0}}^{\xi_{1}} x(t, \xi) \frac{\partial \dot{p}}{\partial \xi}(t, \xi) d \xi=x\left(t, \xi_{1}(t)\right) \dot{p}\left(t, \xi_{1}(t)\right)-x\left(t, \xi_{0}(t)\right) \dot{p}\left(t, \xi_{0}(t)\right)-\int_{\xi_{0}}^{\xi_{1}} \dot{p} \frac{\partial x}{\partial \xi} d \xi= \\
=x\left(t, \xi_{1}(t)\right) \dot{p}\left(t, \xi_{1}(t)\right)-x\left(t, \xi_{0}(t)\right) \dot{p}\left(t, \xi_{0}(t)\right)+\int_{\xi_{0}}^{\xi_{1}} \frac{\partial \Phi}{\partial x} \frac{\partial x}{\partial \xi} d \xi
\end{gathered}
$$

Заметим также, что

$$
\int_{\xi_{0}}^{\xi_{1}} \dot{x} d p(t, \xi)=\int_{\xi_{0}}^{\xi_{1}} \frac{\partial \Phi}{\partial p} \frac{\partial p}{\partial \xi} d \xi
$$

Подставляя два последних равенства в левую часть (23), получаем

$$
\begin{aligned}
\int_{\xi_{0}}^{\xi_{1}}\left(\frac{\partial \Phi}{\partial p} \frac{\partial p}{\partial \xi}+\frac{\partial \Phi}{\partial x} \frac{\partial x}{\partial \xi}\right) d \xi+x\left(t, \xi_{1}\right) \dot{p}\left(t, \xi_{1}\right)-x\left(t, \xi_{0}\right) \dot{p}\left(t, \xi_{0}\right) & = \\
& =\Phi\left(p\left(t, \xi_{1}\right), x_{0}\right)-\Phi\left(p\left(t, \xi_{0}\right), x_{0}\right)+x\left(t, \xi_{1}\right) \dot{p}\left(t, \xi_{1}\right)-x\left(t, \xi_{0}\right) \dot{p}\left(t, \xi_{0}\right)
\end{aligned}
$$

Из последнего равенства и из (23) следует (22), что эквивалентно утверждению теоремы 2.

Заключение. В работе предложено новое определение решения задачи Римана для скалярного закона сохранения с функцией потока, зависящей от $x$ (определение 1 ). Существование и единственность такого решения сразу следуют из того, что введенное в определении множество операторов $S_{t}$ сдвига вдоль траекторий фазового потока ассоциированной гамильтоновой системы является группой относительно операции композиция. 
Однако может оказаться так, что начиная с некоторого момента $t_{0}$ геометрическое решение $S_{t} \gamma$ не является графиком кусочно-непрерывной функции, зависящей от $x$. Для преодоления этой трудности предложена процедура выравнивания, позволяющая по геометрическому решению построить кусочногладкую функцию.

Доказана теорема о корректности определения выравнивания в простейшем случае (теорема 1), сделаны естественные обобщения для того, чтобы расширить класс кривых, к которым применима процедура выравнивания.

В теореме 2 показано, что результат выравнивания геометрического решения, являющегося несложной кривой, для п.в. $t \in[0, T]$ будет кусочногладкой функцией, являющейся обобщенным решением (в смысле $D^{\prime}$ ) исходной задачи Римана.

Кроме того, из раздела о связи условия корректности полного выравнивания с условием допустимости разрыва следует, что в хорошо исследованном в литературе случае $\Phi=\Phi(p)$, т.е. случае, когда функция потока не зависит от $x$, полученное при помощи выравнивания из геометрического решения будет удовлетворять условию Н. Введенской о допустимости разрыва, т.е. будет единственным энтропийным решением соответствующей задачи Римана.

Конкурирующие интересы. Конкурирующих интересов не имею.

Авторская ответственность. Я несу полную ответственность за предоставление окончательной версии рукописи в печать. Окончательная версия рукописи мною одобрена.

Финансирование. Исследование выполнялось без финансирования.

\section{Библиографический список}

1. Bressan A. Hyperbolic systems of conservation laws. The one-dimensional Cauchy problem/ Oxford Lecture Series in Mathematics and its Applications. vol. 20. Oxford: Oxford Univ. Press, 2000. xii +250 pp.

2. Dafermos C. M. Hyperbolic conservation laws in continuum physics / Grundlehren der Mathematischen Wissenschaften. vol. 325. Berlin: Springer, 2006. xxxviii +826 pp. doi: 10.1007/ 978-3-662-49451-6.

3. Годунов С. К., Роменский Е. И. Элементы механики сплошнъх сред и законъ сохранения/ Университетская серия. Т. 4. М.: Научная книга, 1998. 280 с.

4. Evans L. C. Partial differential equations / Graduate Studies in Mathematics. vol. 19. Providence, RI: Amer. Math. Soc., 2010. xxi+749 pp.

5. Colella P., Glaz, H. M. Efficient solution algorithms for the Riemann problem for real gases // J. Comp. Phys., 1985. vol. 59. pp. 264-289. doi: 10.1016/0021-9991(85)90146-9.

6. Lax P. D. Hyperbolic partial differential equations / Courant Lecture Notes in Mathematics. vol. 14. Providence, RI: AMS, 2006. vii +217 pp.

7. Lax P. D., Wendroff B. Systems of conservation laws // Commun. Pure Appl. Math., 1960. vol. 13. pp. 217-237. doi : 10.1002/cpa.3160130205.

8. Кружков С. Н. О задаче Коши в целом для некоторых нелинейных дифференциальных уравнений первого порядка // Докл. АН СССР, 1960. Т. 132, №1. С. 36-39.

9. Субботин А. И. Минимаксные неравенства и уравнения Гамильтона-Якоби. М.: Наука, 1991. 216 с.

10. Колокольцов В. Н., Маслов В. П. Идемпотентный анализ как аппарат теории управления. I// Функи. анализ и его прил., 1989. Т. 23, № 1. С. 1-14.

11. Колокольцов В. Н., Маслов В. П. Идемпотентный анализ как аппарат теории управления и оптимального синтеза. 2 // Функи. анализ и его прил., 1989. Т. 23, № 4. С. 53-62. 
12. Whitham G. B. Linear and nonlinear waves / Pure and Applied Mathematics. A WileyInterscience Series of Texts, Monographs, and Tracts. New York: Wiley, 1999. xvi +636 pp.

13. Danilov V. G., Omel'yanov G. A., Shelkovich V. M Weak asymptotics method and interaction of nonlinear waves / Asymptotic Methods for Wave and Quantum Problems / Amer. Math. Soc. Transl. Ser. 2, 208. Providence, RI: Amer. Math. Soc., 2003. pp. 33165. doi : 10.1090/trans2/208/02.

14. Олейник О. А. О задаче Коши для нелинейных уравнений в классе разрывных функций // Докл. АН ССCP, 1954. Т. 95, № 3. С. 451-454.

15. Введенская Н. Д. Решение задачи Коши для нелинейных уравнений с разрывными начальными условиями методом конечных разностей// Докл. АH CCCP, 1956. Т. 111. C. $517-520$. 
Vestn. Samar. Gos. Tekhn. Univ., Ser. Fiz.-Mat. Nauki

[J. Samara State Tech. Univ., Ser. Phys. Math. Sci.], 2018, vol. 22, no. 4, pp. 620-646

ISSN: 2310-7081 (online), 1991-8615 (print)

doi) http://doi.org/10.14498/vsgtu1634

MSC: 35C99, 35D30, 35L65

\title{
Geometric solutions of the Riemann problem for the scalar conservation law
}

\section{V. Palin}

M. V. Lomonosov Moscow State University, Faculty of Mechanics and Mathematics,

1, Leninskiye Gory, Moscow, 119234, Russian Federation.

\begin{abstract}
For the Riemann problem

$$
\left\{\begin{array}{l}
u_{t}+(\Phi(u, x))_{x}=0 \\
\left.u\right|_{t=0}=u_{-}+[u] \theta(x)
\end{array}\right.
$$

a new definition of the solution is proposed. We associate a Hamiltonian system with initial conservation law, and define the geometric solution as the result of the action of the phase flow on the initial curve. In the second part of this paper, we construct the equalization procedure, which allows us to juxtapose a geometric solution with a unique entropy solution under the condition that $\Phi$ does not depend on $x$. If $\Phi$ depends on $x$, then the equalization procedure allows us to construct a generalized solution of the original Riemann problem.
\end{abstract}

Keywords: Riemann problem, conservation laws, associated Hamiltonian system.

Received: $15^{\text {th }}$ July, $2018 /$ Revised: $11^{\text {th }}$ November, $2018 /$

Accepted: $12^{\text {th }}$ November, $2018 /$ First online: $27^{\text {th }}$ November, 2018

Competing interests. I declare that I have no competing interests.

Author's Responsibilities. I take full responsibility for submitting the final manuscript in print. I approved the final version of the manuscript.

Funding. This research received no specific grant from any funding agency in the public, commercial, or not-for-profit sectors.

\section{Research Article}

()(1) The content is published under the terms of the Creative Commons Attribution 4.0 International License (http://creativecommons.org/licenses/by/4.0/)

Please cite this article in press as:

$\mathrm{P}$ a lin V. V. Geometric solutions of the Riemann problem for the scalar conservation law, Vestn. Samar. Gos. Tekhn. Univ., Ser. Fiz.-Mat. Nauki [J. Samara State Tech. Univ., Ser. Phys. Math. Sci.], 2018, vol. 22, no. 4, pp. 620-646. doi: 10.14498/vsgtu1634 (In Russian).

Author's Details:

Vladimir V. Palin (1) http://orcid.org/0000-0001-7529-7855

Cand. Phys. \& Math. Sci.; Assistant Teacher; Department of Differential Equations;

e-mail: grey_stranger84@mail.ru 


\section{References}

1. Bressan A. Hyperbolic systems of conservation laws. The one-dimensional Cauchy problem, Oxford Lecture Series in Mathematics and its Applications, vol. 20. Oxford, Oxford Univ. Press, 2000, xii +250 pp.

2. Dafermos C. M. Hyperbolic conservation laws in continuum physics, Grundlehren der Mathematischen Wissenschaften, vol. 325. Berlin, Springer, 2006, xxxviii+826 pp. doi: 10.1007/ 978-3-662-49451-6.

3. Godunov S. K., Romenskii E. Elements of Continuum Mechanics and Conservation Laws. New York, Kluwer Acad., 2003, viii+258 pp. doi: 10.1007/978-1-4757-5117-8

4. Evans L. C. Partial differential equations, Graduate Studies in Mathematics, vol. 19. Providence, RI, Amer. Math. Soc., 2010, xxi+749 pp.

5. Colella P., Glaz, H. M. Efficient solution algorithms for the Riemann problem for real gases, J. Comp. Phys., 1985, vol. 59, pp. 264-289. doi: 10.1016/0021-9991(85)90146-9.

6. Lax P. D. Hyperbolic partial differential equations, Courant Lecture Notes in Mathematics, vol. 14. Providence, RI, AMS, 2006, vii +217 pp.

7. Lax P. D., Wendroff B. Systems of conservation laws, Commun. Pure Appl. Math., 1960, vol. 13, pp. 217-237. doi: 10.1002/cpa.3160130205.

8. Kruzkov S. N. The Cauchy problem in the large for certain non-linear first order differential equations, Sov. Math., Dokl., 1960, vol. 1, pp. 474-477.

9. Subbotin A. I. Minimaksnye neravenstva i uravneniia Gamil'tona-Iakobi [Minimax inequalities and Hamilton-Jacobi equations]. Moscow, Nauka, 1991, 216 pp. (In Russian)

10. Kolokol'tsov V. N., Maslov V. P. Idempotent analysis as a tool of control theory and optimal synthesis. I, Funct. Anal. Appl., 1989, vol. 23, no. 1, pp. 1-11. doi: 10.1007/BF01078568.

11. Kolokol'tsov V. N., Maslov V. P. Idempotent analysis as a tool of control theory and optimal synthesis. 2, Funct. Anal. Appl., 1989, vol. 23, no. 4, pp. 300-307. doi : 10.1007/BF01078944.

12. Whitham G. B. Linear and nonlinear waves, Pure and Applied Mathematics. A WileyInterscience Series of Texts, Monographs, and Tracts. New York, Wiley, 1999, xvi+636 pp.

13. Danilov V. G., Omel'yanov G. A., Shelkovich V. M Weak asymptotics method and interaction of nonlinear waves, In: Asymptotic Methods for Wave and Quantum Problems, Amer. Math. Soc. Transl. Ser. 2, 208. Providence, RI, Amer. Math. Soc., 2003, pp. 33-165. doi: 10.1090/trans2/208/02.

14. Oleinik O. A. On the Cauchy problem for nonlinear equations in the class of discontinuous functions, Dokl. Akad. Nauk SSSR, 1954, vol.95, no. 3, pp. 451-454 (In Russian).

15. Vvedenskaya N. D. Solution of the Cauchy problem for a nonlinear equation with discontinuous initial conditions by the method of finite differences, Dokl. Akad. Nauk SSSR, 1956, vol. 111, pp. 517-520 (In Russian). 